\title{
The Central Limit Theorem for uniformly strong mixing measures
}

\author{
Nicolai Haydn*
}

October 25, 2018

\begin{abstract}
The theorem of Shannon-McMillan-Breiman states that for every generating partition on an ergodic system, the exponential decay rate of the measure of cylinder sets equals the metric entropy almost everywhere (provided the entropy is finite). In this paper we prove that the measure of cylinder sets are lognormally distributed for strongly mixing systems and infinite partitions and show that the rate of convergence is polynomial provided the fourth moment of the information function is finite. Also, unlike previous results by Ibragimov and others which only apply to finite partitions, here we do not require any regularity of the conditional entropy function. We also obtain the law of the iterated logarithm and the weak invariance principle for the information function.
\end{abstract}

\section{Introduction}

Let $\mu$ be a $T$-invariant probability measure on a space $\Omega$ on which the map $T$ acts measurably. For a measurable partition $\mathcal{A}$ one forms the $n$th join $\mathcal{A}^{n}=\bigvee_{j=0}^{n-1} T^{-j} \mathcal{A}$ which forms a finer partition of $\Omega$. (The atoms of $\mathcal{A}^{n}$ are traditionally called $n$-cylinders.) For $x \in \Omega$ we denote by $A_{n}(x) \in \mathcal{A}^{n}$ the $n$-cylinder which contains $x$. The Theorem of Shannon-McMillan-Breiman (see e.g. [23, 30]) then states that for $\mu$-almost every $x$ in $\Omega$ the limit

$$
\lim _{n \rightarrow \infty} \frac{-\log \mu\left(A_{n}(x)\right)}{n}
$$

exists and equals the metric entropy $h(\mu)$ provided the entropy is finite in the case of a countable infinite partition. It is easy to see that this convergence is not uniform (not even for Bernoulli measures with weights that are not all equal). This theorem was proved for finite partitions in increasing degrees of generality in the years 1948 to 1957 and then was by Carleson [8] and Chung [10] generalised to infinite partitions. Similar results (for finite partitions) for the recurrence and waiting times were later proved by Ornstein and Weiss [26] and Nobel and Wyner [25] respectively. The limiting behaviour for recurrence times was generalised in 2002 by Ornstein and Weiss [27] to countably infinite partitions. In the present paper we are concerned with the limiting distribution of the information function $I_{n}(x)=-\log \mu\left(A_{n}(x)\right)$ around its mean value.

The statistical properties of $I_{n}$ are of great interest in information theory where they are connected to the efficiency of compression schemes. Let us also note that in dynamical systems the analog of SMB's theorem for compact metric spaces is the Brin-Katok local entropy formula [5] which states that for ergodic invariant measures the exponential decay rate of dynamical balls is almost everywhere equal to the entropy.

There is a large classical body of work on the Central Limit Theorem (CLT) for independent random variables. For dependent random variables the first CLTs are due to Markov (for Markov chains) and Bernstein [2] for random variables that are allowed to have some short range dependency but have to be independent if separated by a suitable time difference (for more than a power of the length $n$ of the partial sums $S_{n}$ ). In 1956 Rosenblatt [35] then introduced the notions of uniform mixing and strong

*Mathematics Department, USC, Los Angeles, 90089-1113. E-mail: <nhaydn@math.usc.edu>. This work was supported by a grant from the NSF (DMS-0301910). 
mixing (see below) and proved a CLT for the partial sums $S_{n}$ of random variables that satisfy the strong mixing property. In 36, he then proved a more general CLT for random variables on systems that satisfy an $L^{2}$ norm condition 1 . Around the same time Nagaev [24] proved a convergence theorem for the stable law for strongly mixing systems. His result covers the case of the CLT and formed the basis for Ibragimov's famous 1962 paper [20] in which he proved for finite partitions 'a refinement to SMB's theorem' by showing that $I_{n}(x)=-\log \mu\left(A_{n}(x)\right)$ is in the limit lognormally distributed for systems that are strongly mixing and satisfy a regularity condition akin to a Gibbs property. Based on his results and methods, Philipp and Stout [32] proved the almost sure invariance principle for the information function $I_{n}$ under similar conditions as Ibragimov used (requiring faster decay rates). This result in turn was then used by Kontoyiannis 21] to prove the almost sure invariance principle, CLT and the law of the iterated logarithm LIL for recurrence and waiting times, thus strengthening the result of Nobel and Wyner 25. who showed that for strongly mixing systems (without regularity condition) the exponential growth rate of waiting times equals the metric entropy.

Various improvements and refinements to the CLT for the information function have been successively done mainly for measures that satisfy a genuine Gibbs property. For instance Collet, Galves and Schmitt 12 in order to prove the lognormal distribution of entry times for exponentially $\psi$-mixing Gibbs measure 2 needed to know that $I_{n}$ is in the limit lognormally distributed. A more general result is due to Paccaut [28] for maps on the interval where he had to assume some topological covering properties. For some non-uniformly hyperbolic maps on the interval similar results were formulated in [14, 7]. However all those results use explicitly the Gibbs property of the invariant measure $\mu$ to approximate the information function $I_{n}$ by an ergodic sum and then to invoke standard results on the CLT for sufficiently regular observables (see for instance [17, 22, 9]). (Of course the variance has to be non-zero because otherwise the limiting distribution might not be normal as an example in [12] illustrates.)

Results that do not require the explicit Gibbs characterisation of the measure like Kontoyiannis' paper [21, all ultimately rely on the original paper of Ibragimov [20] and require apart from the strong mixing condition the regularity of the Radon-Nikodym derivative of the measure under the local inverse maps. In [18 we went beyond his regularity constraint and proved a CLT with error bounds for the lognormal distribution of the information function for $(\psi, f)$-mixing systems which included traditional $\psi$-mixing maps and also equilibrium states for rational maps with critical points in the Julia set.

The present paper is significant in two respects: (i) we allow for the partition to be countably infinite instead of finite and (ii) unlike Ibragimov (and all who followed him) we do not require an $L^{1}$-regularity condition for the Radon Nikodym derivative for local inverses of the map. This condition which was introduced in [20] is the $L^{1}$ equivalent of what otherwise would allow a transfer operator approach to analyse the invariant measure and imply the Gibbs property 3 . We moreover prove that the rate of convergence is polynomial (Theorem 2) and the variance is always positive for genuinely infinite partitions.

Let us note that convergence rates for the CLT have previously been obtained by A Broise [6] for a large class of expanding maps on the interval for which the Perron-Frobenius operator has a 'spectral gap'. Similar estimates were obtained by Pène [29] for Gibbs measures for dispersing billiards.

This paper is structured as follows: In the second section we introduce uniform strong mixing systems and in the third section we prove the existence of the variance $\sigma^{2}$ of strongly mixing probability measures (Proposition 14) as well as the growth rate of higher order moments (Proposition [15). This is the main part of the proof (note that Ibragimov's regularity condition was previously needed precisely to obtain the variance of the measure). In section 4 we then prove the CLT using Stein's method of exchangeable

\footnotetext{
1 The map $T$ satisfies an $L^{2}$ norm condition if $\sup _{f: \mu(f)=0} \frac{\left\|T^{n} f\right\|^{2}}{\|f\|^{2}}$ decays exponentially fast as $n \rightarrow \infty$. This is a somewhat stronger mixing condition than the strong mixing condition

${ }^{2}$ We say an invariant probability measures $\mu$ is Gibbs for a potential $f$ with pressure $P(f)$ if there exists a constant $c>0$ so that

$$
\frac{1}{c} \leq \frac{\mu\left(A_{n}(x)\right)}{e^{f(x)+f(T x)+\cdots+f\left(T^{n-1} x\right)-n P(f)}} \leq c
$$

for every $x \in \Omega$ and $n=1,2, \ldots$.

3 More precisely, Ibragimov's condition requires that the $L^{1}$-norms of the differences $f-f_{n}$ decay polynomially, where $f=\lim _{n \rightarrow \infty} f_{n}$ and $f_{n}(x)=\log \mathbb{P}\left(x_{0} \mid x_{-1} x_{-2} \ldots x_{-n}\right)$.
} 
pairs. In section 5 we prove the Weak Invariance Principle for $I_{n}$ using the CLT and the convergence rate obtained in section 3 .

I would like to thank my colleague Larry Goldstein for many conversations in which he explained Stein's method to me.

\section{Main results}

Let $T$ be a map on a space $\Omega$ and $\mu$ a probability measure on $\Omega$. Moreover let $\mathcal{A}$ be a (possibly infinite) measurable partition of $\Omega$ and denote by $\mathcal{A}^{n}=\bigvee_{j=0}^{n-1} T^{-j} \mathcal{A}$ its $n$-th join which also is a measurable partition of $\Omega$ for every $n \geq 1$. The atoms of $\mathcal{A}^{n}$ are called $n$-cylinders. Let us put $\mathcal{A}^{*}=\bigcup_{n=1}^{\infty} \mathcal{A}^{n}$ for the collection of all cylinders in $\Omega$ and put $|A|$ for the length of a cylinder $A \in \mathcal{A}^{*}$, i.e. $|A|=n$ if $A \in \mathcal{A}^{n}$.

We shall assume that $\mathcal{A}$ is generating, i.e. that the atoms of $\mathcal{A}^{\infty}$ are single points in $\Omega$.

\subsection{Mixing}

Definition 1 We say the invariant probability measure $\mu$ is uniformly strong mixing if there exists a decreasing function $\psi: \mathbb{N} \rightarrow \mathbb{R}^{+}$which satisfies $\psi(\Delta) \rightarrow 0$ as $\Delta \rightarrow \infty$ so that

$$
\left|\sum_{(B, C) \in S}(\mu(B \cap C)-\mu(B) \mu(C))\right| \leq \psi(\Delta)
$$

for every subset $S$ of $\mathcal{A}^{n} \times T^{-\Delta-n} \mathcal{A}^{m}$ and every $n, m, \Delta>0$.

\section{Various kinds of mixing:4}

In the following list of different mixing properties $U$ is always in the $\sigma$-algebra generated by $\mathcal{A}^{n}$ and $V$ lies in the $\sigma$-algebra generated by $\mathcal{A}^{*}$ (see also [13]). The limiting behaviour is as the length of the 'gap' $\Delta \rightarrow \infty$ :

1. $\psi$-mixing: $\sup _{n} \sup _{U, V}\left|\frac{\mu\left(U \cap T^{-\Delta-n} V\right)}{\mu(U) \mu(V)}-1\right| \rightarrow 0$.

2. Left $\phi$-mixing: $\sup _{n} \sup _{U, V}\left|\frac{\mu\left(U \cap T^{-\Delta-n} V\right)}{\mu(U)}-\mu(V)\right| \rightarrow 0$.

3. Strong mixing [35, 20] (also called $\alpha$-mixing): $\sup _{n} \sup _{U, V}\left|\mu\left(U \cap T^{-\Delta-n} V\right)-\mu(U) \mu(V)\right| \rightarrow 0$.

4. Uniform mixing [35, 36]: $\sup _{n} \sup _{U, V}\left|\frac{1}{k} \sum_{j=1}^{k} \mu\left(U \cap T^{-n-j} V\right)-\mu(U) \mu(V)\right| \rightarrow 0$ as $k \rightarrow \infty$.

One can also have right $\phi$-mixing when $\sup _{n} \sup _{U, V}\left|\frac{\mu\left(U \cap T^{-\Delta-n} V\right)}{\mu(V)}-\mu(U)\right| \rightarrow 0$ as $\Delta \rightarrow \infty$. Clearly $\psi$-mixing implies all the other kinds of mixing. The next strongest mixing property is $\phi$-mixing, then comes strong mixing and uniform mixing is the weakest. The uniform strong mixing property is stronger that the strong mixing property but is implies by the dynamical $\phi$-mixing property as we will see in Lemma 6. In fact if $\mu$ is strong mixing then the sets $S$ in Definition 1 have to be of product form.

For a partition $\mathcal{A}$ we have the ( $n$-th) information function $I_{n}(x)=-\log \mu\left(A_{n}(x)\right)$, where $A_{n}(x)$ denotes the unique $n$-cylinder that contains the point $x \in \Omega$, whose moments are

$$
K_{w}(\mathcal{A})=\sum_{A \in \mathcal{A}} \mu(A)|\log \mu(A)|^{w}=\mathbb{E}\left(I_{n}^{w}\right),
$$

\footnotetext{
${ }^{4}$ Here we adopt probabilistic terminology which differs from the one used in the dynamical systems community.
} 
$w \geq 0$ not necessarily integer. (For $w=1$ one traditionally writes $H(\mathcal{A})=K_{1}(\mathcal{A})=\sum_{A \in \mathcal{A}}-\mu(A) \log \mu(A)$.) If $\mathcal{A}$ is finite then $K_{w}(\mathcal{A})<\infty$ for all $w$. For infinite partitions the theorem of Shannon-McMillan-Breiman requires that $H(\mathcal{A})$ be finite $[8,10$. In order to prove that the information function is lognormally distributed we will require a larger than fourth moment $K_{w}(\mathcal{A})$ for some $w>4$ (not necessarily integer) be finite.

\subsection{Results}

For $x \in \Omega$ we denote $A_{n}(x)$ the $n$-cylinder in $\mathcal{A}^{n}$ which contains the point $x$. We are interested in the limiting behaviour of the distribution function

$$
\Xi_{n}(t)=\mu\left(\left\{x \in \Omega: \frac{-\log \mu\left(A_{n}(x)\right)-n h}{\sigma \sqrt{n}} \leq t\right\}\right)
$$

for real valued $t$ and a suitable positive $\sigma$, where $h$ is the metric entropy of $\mu$. The Central Limit Theorem states that this quantity converges to the normal distribution $N(t)=\frac{1}{\sqrt{2 \pi}} \int_{-\infty}^{t} e^{-s^{2} / 2} d s$ as $n$ goes to infinity if there exists a suitable $\sigma$ which is positive. Our main result is the following theorem:

Theorem 2 Let $\mu$ be a uniformly strong mixing probability measure on $\Omega$ with respect to a countably finite, measurable and generating partition $\mathcal{A}$ which satisfies $K_{w}(\mathcal{A})<\infty$ for some $w>4$. Assume that $\psi$ decays at least polynomially with power $>8+\frac{24}{w-4}$.

Then

(I) The limit

$$
\sigma^{2}=\lim _{n \rightarrow \infty} \frac{K_{2}\left(\mathcal{A}^{n}\right)-H^{2}\left(\mathcal{A}^{n}\right)}{n}
$$

exists and defines the variance of $\mu$. Moreover if the partition is infinite then $\sigma$ is strictly positive. (II) If $\sigma>0$ :

$$
\left|\Xi_{n}(t)-N(t)\right| \leq C_{0} \frac{1}{n^{\kappa}}
$$

for all $t$ and all

(i) $\kappa<\frac{1}{10}-\frac{3}{5} \frac{w}{(p+2)(w-2)+6}$ if $\psi$ decays polynomially with power $p$,

(ii) $\kappa<\frac{1}{10}$ if $\psi$ decays faster than any power.

The variance $\sigma^{2}$ is determined in Proposition 14 and essentially only requires finiteness of the second moment $K_{2}(\mathcal{A})$. In order to obtain the rate of convergence one usually needs a higher than second moment of $I_{n}$. Since we use Stein's method we require the fourth moment be finite (unlike in [18] where for finite partitions and $(\psi, f)$-mixing measures we only needed bounds on the third moment).

Throughout the paper we shall assume that $K_{w}(\mathcal{A})<\infty$ for some finite $w>4$. The case in which $w$ can be arbitrarily large (e.g. for finite partitions) is done with minor modifications and yields the obvious result for the rate of convergence. For simplicity's sake we assume in the proofs that the decay rate of $\psi$ is polynomial at some finite power $p$. The case of hyper polynomial decay can be traced out with minor modifications and yields the stated result.

If the partition $\mathcal{A}$ is finite then $K_{w}(\mathcal{A})<\infty$ for all $w$ and we obtain the following corollary:

Corollary 3 Let $\mu$ be a uniformly strong mixing probability measure on $\Omega$ with respect to a finite, measurable and generating partition $\mathcal{A}$ and $\psi$ decays at least polynomially with power $>8+\frac{24}{w-4}$.

Then

(I) The limit $\sigma^{2}=\lim _{n \rightarrow \infty} \frac{1}{n}\left(K_{2}\left(\mathcal{A}^{n}\right)-H^{2}\left(\mathcal{A}^{n}\right)\right)$ exists (variance of $\mu$ ).

(II) If $\sigma>0: \Xi_{n}(t)=N(t)+\mathcal{O}\left(n^{-\kappa}\right)$ for all $t$ and $\left\{\begin{array}{l}\kappa<\frac{1}{10}-\frac{3}{5(p+2)} \text { if } \psi(\Delta)=\mathcal{O}\left(\Delta^{-p}\right), \Delta \in \mathbb{N} \text {, } \\ \kappa<\frac{1}{10} \text { if } \psi \text { decays hyper polynomially. }\end{array}\right.$

By a result of Petrov [31] we now obtain the Law of the Iterated Logarithm from Theorem 2 by virtue of the error bound (better than $\frac{1}{(\log n)^{1-\varepsilon}}($ some $\varepsilon>0)$ which are the ones required in [31]). 
Corollary 4 Under the assumptions of Theorem 2:

$$
\limsup _{n \rightarrow \infty} \frac{I_{n}(x)-n h}{\sigma \sqrt{2 n \log \log n}}=1
$$

almost everywhere.

A similar statement is true for the liminf where the limit is then equal to -1 almost everywhere.

Based on the Central Limit Theorem we also get the weak invariance principle WIP (see section 4). Recently there has been a great interest in the WIP in relation to mixing properties of dynamical systems. For instance it has been obtained for a large class of observables and for a large class of dynamical systems by Chernov in 9 . Other recent results are [15, 14, 32. Those results however are typically for sums of sufficiently regular observables. Here we prove the WIP for $I_{n}(x)$.

Theorem 5 Under the assumption of Theorem 圆 the information function $I_{n}$ satisfies the Weak Invariance Principle (provided the variance $\sigma^{2}$ is positive).

\subsection{Examples}

(I) Bernoulli shift: Let $\Sigma$ be the full shift space over the infinite alphabet $\mathbb{N}$ and let $\mu$ be the Bernoulli measure generated by the positive weights $p_{1}, p_{2}, \ldots\left(\sum_{j} p_{j}=1\right)$. The entropy is then $h(\mu)=\sum_{j} p_{j}\left|\log p_{j}\right|$ and since $K_{2}(\mathcal{A})=\sum_{i} p_{i} \log ^{2} p_{i}=\frac{1}{2} \sum_{i, j} p_{i} p_{j}\left(\log ^{2} p_{i}+\log ^{2} p_{j}\right)$ we obtain that the variance is given by the following expression which is familiar from finite alphabet Bernoulli shifts:

$$
\sigma^{2}=K_{2}(\mathcal{A})-h(\mu)^{2}=\frac{1}{2} \sum_{i, j} p_{i} p_{j} \log ^{2} \frac{p_{i}}{p_{j}}
$$

We have used that the partition $\mathcal{A}$ is given by the cylinder sets whose first symbols are fixed. Here we naturally assume that $\sum_{i} p_{i} \log ^{2} p_{i}<\infty$. If moreover $\sum_{i} p_{i} \log ^{4} p_{i}<\infty$ then

$$
\mathbb{P}\left(\frac{-\log \mu\left(A_{n}(x)\right)-n h}{\sigma \sqrt{n}} \leq t\right)=N(t)+\mathcal{O}\left(r^{-1 / 4}\right)
$$

with exponent $\frac{1}{4}$ which is a well known result for unbounded iid random variables. With other techniques one can however weaken the moment requirement in this case.

(II) Markov shift: Again let $\Sigma$ be the shift space over the infinite alphabet $\mathbb{N}$ and $\mu$ the Markov measure generated by an infinite probability vector $\vec{p}=\left(p_{1}, p_{2}, \ldots\right)\left(p_{j}>0, \sum_{j} p_{j}=1\right)$ and an infinite stochastic matrix $P(\vec{p} P=\vec{p}, P \mathbf{1}=\mathbf{1})$. The partition $\mathcal{A}$ is again the partition of single element cylinder sets. If $\vec{x}=x_{1} x_{2} \ldots x_{n}$ is a word of length $n$ (we write $\vec{x} \in \mathcal{A}^{n}$ ) then the measure of its cylinder set is $\mu(\vec{x})=p_{x_{1}} P_{x_{1} x_{2}} P_{x_{2} x_{3}} \cdots P_{x_{n-1} x_{n}}$. The metric entropy is $h(\mu)=\sum_{i, j}-p_{i} P_{i j} \log P_{i j}$ [38] and the variance [34, 40] (see also Appendix) is

$$
\sigma^{2}=\frac{1}{2} \sum_{i j k \ell} p_{i} P_{i j} p_{k} P_{k \ell} \log ^{2} \frac{P_{i j}}{P_{k \ell}}+4 \sum_{k=2}^{\infty} \sum_{\vec{x} \in \mathcal{A}^{k}} \mu(\vec{x})\left(\log P_{x_{1} x_{2}} \log P_{x_{k-1} x_{k}}-h^{2}\right) .
$$

(III) Gibbs states: The measure $\mu$ is a Gibbs state for the potential function $f$ if there exists a constant $c>1$ so that for every point $x \in \Omega$ and $n$ one has $\mu\left(A_{n}(x)\right) \in\left[\frac{1}{c}, c\right] e^{f^{n}(x)-n P(f)}$ where $P(f)$ is the pressure of $f$ and $f^{n}=f+f \circ T+\cdots+f \circ T^{n-1}$ is the $n$th ergodic sum of $f$. If $f$ is Hölder continuous and $T$ is and Axiom A map, then $\mu$ is the unique equilibrium state. In this case the CLT has been studied a great deal in particular for finite partitions since standard techniques for sums of random variables can be applied (see e.g. 6, 17, 22, 29]). Note that (I) and (II) are special cases of Gibbs states. 


\section{Variance and higher moments}

\subsection{Some basic properties}

Let us begin by showing that the uniform strong mixing property is implied by the $\phi$-mixing property.

Lemma 6 -mixing implies uniformly strong mixing.

Proof. Let $\mu$ be a left $\phi$-mixing probability measure (the right $\phi$-mixing case is done in the same way). That means, there exists a decreasing $\phi(\Delta) \rightarrow 0$ as $\Delta \rightarrow \infty$ so that

$$
|(\mu(B \cap C)-\mu(B) \mu(C))| \leq \phi(\Delta) \mu(B)
$$

for every $C$ in the $\sigma$-algebra generated by $\mathcal{C}=T^{-n-\Delta} \mathcal{A}^{m}$ and every cylinder $B \in \mathcal{B}=\mathcal{A}^{n}$ for all $n$ and $\Delta$. Let $S \subset \mathcal{B} \times \mathcal{C}$ and put $S_{B}$ for the interection of $\{B\} \times \mathcal{C}$ with $S$. Then $\left|\left(\mu\left(B \cap S_{B}\right)-\mu(B) \mu\left(S_{B}\right)\right)\right| \leq$ $\phi(\Delta) \mu(B)$ and

$$
\left|\sum_{(B, C) \in S}(\mu(B \cap C)-\mu(B) \mu(C))\right| \leq \sum_{B \in \mathcal{B}}\left|\left(\mu\left(B \cap S_{B}\right)-\mu(B) \mu\left(S_{B}\right)\right)\right| \leq \sum_{B \in \mathcal{B}} \phi(\Delta) \mu(B) \leq \phi(\Delta)
$$

implies that $\mu$ is uniformly strong mixing with $\psi=\phi$.

The following estimate has previously been shown for $\psi$-mixing measures (in which case they are exponential) in [16] and for $\phi$-mixing measures in [1]. Denote by $A_{n}(x)$ the atom in $\mathcal{A}^{n}(n=1,2, \ldots)$ which contains the point $x \in \Omega$. (Abadi [1] also showed that in case (II) the decay cannot in general be exponential.)

Lemma 7 Let $\mu$ be strong mixing. Then there exists a constant $C_{1}$ so that for all $A \in \mathcal{A}_{n}, n=1,2, \ldots$ : (I) $\mu(A) \leq C_{1} n^{-p}$ if $\psi$ is polynomially decreasing with exponent $p>0$;

(II) $\mu(A) \leq C_{1} \theta^{\sqrt{n}}$ for some $\theta \in(0,1)$ if $\psi$ is exponentially decreasing.

Proof. Fix $m \geq 1$ so that $a=\max _{A \in \mathcal{A}^{m}} \mu(A)$ is less than $\frac{1}{4}$ and let $\Delta_{1}, \Delta_{2}, \ldots$ be integers which will be determined below. We put $n_{j}=j m+\sum_{i=1}^{j-1} \Delta_{i}$ (put $\Delta_{0}=0$ ) and for $x \in \Omega$ let $B_{j}=A_{m}\left(T^{n_{j-1}+m} x\right.$ ) and put $C_{k}=\bigcap_{j=1}^{k} B_{j}$. Then $A_{n_{k}}(x) \subset C_{k}$ and

$$
\mu\left(C_{k+1}\right)=\mu\left(C_{k} \cap B_{k+1}\right)=\mu\left(C_{k}\right) \mu\left(B_{k+1}\right)+\rho\left(C_{k}, B_{k+1}\right)
$$

where the remainder term $\rho\left(C_{k}, B_{k+1}\right)$ is by the mixing property in absolute value bounded by $\psi\left(\Delta_{k}\right)$. Now we choose $\Delta_{j}$ so that $\psi\left(\Delta_{j}\right) \leq a^{\frac{j}{2}+1}$. Then $\mu\left(C_{k+1}\right) \leq \mu\left(C_{k}\right) a+a^{\frac{k}{2}+1}$ implies that $\mu\left(C_{k}\right) \leq c_{0} a^{\frac{k}{2}}$ (as $\sqrt{a} \leq \frac{1}{2}$ ) for some $c_{0}>0$.

(I) If $\psi$ decays polynomially with power $p$, i.e. $\psi(t) \leq c_{1} t^{-p}$, then the condition $\psi\left(\Delta_{j}\right) \leq a^{\frac{j}{2}+1}$ is satisfied if we put $\Delta_{j}=\left[c_{2} a^{-\frac{j}{2 p}}\right]$ for a suitable constant $c_{2}>0$. Consequently $n_{k} \leq c_{3} a^{-\frac{k}{2 p}}\left(c_{3} \geq 1\right)$ and therefore $k \geq 2 p \frac{\log n_{k}}{|\log a|}$. Hence

$$
\mu\left(A_{n_{k}}(x)\right) \leq c_{0} a^{\frac{k}{2}} \leq c_{0} a^{p \frac{\log n_{k}}{\log a}} \leq c_{4} n_{k}^{-p}
$$

and from this one obtains $\mu\left(A_{n}(x)\right) \leq c_{5} n^{-p}$ for all integers $n$ (and some larger constant $c_{5}$ ).

(II) If $\psi$ decays exponentially, i.e. $\psi(t) \leq c_{6} \vartheta^{t}$ for some $\vartheta \in(0,1)$, then we choose $\Delta_{j}=\left[\frac{j}{2} \frac{\log a}{\log \vartheta}\right]$ and obtain $n_{k} \leq m k+c_{7} k^{2}$, which gives us $k \geq c_{8} \sqrt{n_{k}}\left(c_{8}>0\right)$ and the stretched exponential decay of the measure of cylindersets:

$$
\mu\left(A_{n}(x)\right) \leq c_{9} a^{c_{8} \sqrt{n}} .
$$

Now put $\theta=a^{c 8}$. 


\subsection{The information function and mixing properties}

The metric entropy $h$ for the invariant measure $\mu$ is $h=\lim _{n \rightarrow \infty} \frac{1}{n} H\left(\mathcal{A}^{n}\right)$, where $\mathcal{A}$ is a generating partition of $\Omega$ (cf. [23]), provided $H(\mathcal{A})<\infty$. For $w \geq 1$ put $\eta_{w}(t)=t \log ^{w} \frac{1}{t}\left(\eta_{w}(0)=0\right)$. Then

$$
K_{w}(\mathcal{B})=\sum_{B \in \mathcal{B}} \mu(B)|\log \mu(B)|^{w}=\sum_{B \in \mathcal{B}} \eta_{w}(\mu(B))
$$

for partitions $\mathcal{B}$. Similarly one has the conditional quantity $(\mathcal{C}$ is a partition):

$$
K_{w}(\mathcal{C} \mid \mathcal{B})=\sum_{B \in \mathcal{B}, C \in \mathcal{C}} \mu(B) \eta_{w}\left(\frac{\mu(B \cap C)}{\mu(B)}\right)=\sum_{B, C} \mu(B \cap C)\left|\log \frac{\mu(B \cap C)}{\mu(B)}\right|^{w} .
$$

Lemma 8 [18] For any two partitions $\mathcal{B}, \mathcal{C}$ for which $K_{w}(\mathcal{B}), K_{w}(\mathcal{C})<\infty$ and $\mu(C) \leq e^{-w} \forall C \in \mathcal{C}$ :

(i) $K_{w}(\mathcal{C} \mid \mathcal{B}) \leq K_{w}(\mathcal{C})$,

(ii) $K_{w}(\mathcal{B} \vee \mathcal{C})^{1 / w} \leq K_{w}(\mathcal{C} \mid \mathcal{B})^{1 / w}+K_{w}(\mathcal{B})^{1 / w}$

(iii) $K_{w}(\mathcal{B} \vee \mathcal{C})^{1 / w} \leq K_{w}(\mathcal{C})^{1 / w}+K_{w}(\mathcal{B})^{1 / w}$

Proof. (i) Since $\eta_{w}(t)$ is convex and increasing on $\left[0, e^{-w}\right]$ and decreasing to zero on $\left(e^{-w}, 1\right]$ we have $\sum_{i} x_{i} \eta_{w}\left(\alpha_{i}\right) \leq \eta_{w}\left(\sum_{i} x_{i} \alpha_{i}\right)$ for weights $x_{i} \geq 0\left(\sum_{i} x_{i}=1\right)$ and numbers $\alpha_{i} \in[0,1]$ which satisfy $\sum_{i} x_{i} \alpha_{i} \leq e^{-w}$. Hence

$$
K_{w}(\mathcal{C} \mid \mathcal{B})=\sum_{B \in \mathcal{B}, C \in \mathcal{C}} \mu(B) \eta_{w}\left(\frac{\mu(B \cap C)}{\mu(B)}\right) \leq \sum_{C} \eta_{w}\left(\sum_{B} \mu(B) \frac{\mu(B \cap C)}{\mu(B)}\right)=\sum_{C} \eta_{w}(\mu(C))=K_{w}(\mathcal{C}) .
$$

(ii) The second statement follows from Minkowski's inequality on $L^{w}$-spaces:

$$
\begin{aligned}
K_{w}(\mathcal{B} \vee \mathcal{C})^{\frac{1}{w}} & =\left(\sum_{B \in \mathcal{B}, C \in \mathcal{C}} \mu(B \cap C)|\log \mu(B \cap C)|^{w}\right)^{\frac{1}{w}} \\
& \leq\left(\sum_{B, C} \mu(B \cap C)\left|\log \frac{\mu(B \cap C)}{\mu(B)}\right|^{w}\right)^{\frac{1}{w}}+\left(\sum_{B, C} \mu(B \cap C)|\log \mu(B)|^{w}\right)^{\frac{1}{w}} \\
& =K_{w}(\mathcal{C} \mid \mathcal{B})^{\frac{1}{w}}+K_{w}(\mathcal{B})^{\frac{1}{w}} .
\end{aligned}
$$

(iii) This follows from (ii) and (i).

Corollary 9 Let $w \geq 1$ and $\mathcal{A}$ so that $K_{w}(\mathcal{A})<\infty$ and $\mu(A) \leq e^{-w} \forall A \in \mathcal{A}$. Then there exists a constant $C_{2}$ (depending on $w$ ) so that for all $n$

$$
K_{w}\left(\mathcal{A}^{n}\right) \leq C_{2} n^{w} .
$$

Proof. We want to use Lemma 8 (iii) to show that the sequence $a_{n}=K_{w}\left(\mathcal{A}^{n}\right)^{1 / w}, n=1,2, \ldots$, is subadditive. The hypothesis of Lemma 8 is satisfied since $\mu(A) \leq e^{-w}$ for all $A \in \mathcal{A}$. We thus obtain $K_{w}\left(\mathcal{A}^{n+m}\right)^{\frac{1}{w}} \leq K_{w}\left(\mathcal{A}^{n}\right)^{\frac{1}{w}}+K_{w}\left(\mathcal{A}^{m}\right)^{\frac{1}{w}}$ for all $n, m \geq 1$ and therefore subadditivity of the sequence $a_{n}$. Since by assumption $K_{w}(\mathcal{A})<\infty$ we get that the $\operatorname{limit}_{n \rightarrow \infty} \frac{1}{n} K_{w}\left(\mathcal{A}^{n}\right)^{1 / w}$ exists, is finite and equals the inf (see e.g. [38]).

The function $I_{n}$ has expected value $\mathbb{E}\left(I_{n}\right)=H\left(\mathcal{A}^{n}\right)$, for which we also write $H_{n}$, and variance $\sigma_{n}^{2}=$ $\sigma^{2}\left(I_{n}\right)=K_{2}\left(\mathcal{A}^{n}\right)-H_{n}^{2}$. In general, if $\mathcal{B}$ is a partition then we write $\sigma^{2}(\mathcal{B})=K_{2}(\mathcal{B})-H^{2}(\mathcal{B})$ and similarly for the conditional variance $\sigma^{2}(\mathcal{C} \mid \mathcal{B})$. Let us define the function $J_{\mathcal{B}}$ by $J_{\mathcal{B}}(B)=-\log \mu(B)-H(\mathcal{B})(B \in \mathcal{B})$ then $\sigma^{2}(\mathcal{B})=\sum_{B \in \mathcal{B}} \mu(B) J_{\mathcal{B}}(B)^{2}$ and $\int J_{\mathcal{B}} d \mu=0$. For two partitions $\mathcal{B}$ and $\mathcal{C}$ we put

$$
J_{\mathcal{C} \mid \mathcal{B}}(B \cap C)=\log \frac{\mu(B)}{\mu(B \cap C)}-H(\mathcal{C} \mid \mathcal{B})
$$

for $(B, C) \in \mathcal{B} \times \mathcal{C}$. (This means $J_{\mathcal{C} \mid \mathcal{B}}=J_{\mathcal{B} \vee \mathcal{C}}-J_{\mathcal{B}}$ and $\sigma(\mathcal{C} \mid \mathcal{B})=\sigma\left(J_{\mathcal{C} \mid \mathcal{B}}\right)$.) 
Lemma 10 Let $\mathcal{B}$ and $\mathcal{C}$ be two partitions. Then

$$
\sigma(\mathcal{B} \vee \mathcal{C}) \leq \sigma(\mathcal{C} \mid \mathcal{B})+\sigma(\mathcal{B})
$$

Proof. This follows from Minkowski's inequality

$$
\sigma(\mathcal{B} \vee \mathcal{C})=\sqrt{\mu\left(J_{\mathcal{C} \mid \mathcal{B}}+J_{\mathcal{B}}\right)^{2}} \leq \sqrt{\mu\left(J_{\mathcal{C} \mid \mathcal{B}}^{2}\right)}+\sqrt{\mu\left(J_{\mathcal{B}}^{2}\right)}=\sigma(\mathcal{C} \mid \mathcal{B})+\sigma(\mathcal{B})
$$

As a consequence of Lemma $8(\mathrm{i})$ one also has $K_{w}(\mathcal{B} \vee \mathcal{C} \mid \mathcal{B})=K_{w}(\mathcal{C} \mid \mathcal{B}) \leq K_{w}(\mathcal{C})$ which in particular implies $\sigma(\mathcal{B} \vee \mathcal{C} \mid \mathcal{B})=\sigma(\mathcal{C} \mid \mathcal{B}) \leq \sqrt{K_{2}(\mathcal{C})}$. As before we put $\rho(B, C)=\mu(B \cap C)-\mu(B) \mu(C)$ (and in the following we often write $\mathcal{B}=\mathcal{A}^{n}$ and $\mathcal{C}=T^{-\Delta-n} \mathcal{A}^{n}$ for integers $\left.n, \Delta\right)$.

The following technical lemma is central to get the variance of $\mu$ and bounds on the higher moments of $J_{n}=I_{n}-H_{n}$.

Lemma 11 Let $\mu$ be uniformly strong mixing and assume that $K_{w}(\mathcal{A})<\infty$ and $\mu(A) \leq e^{-w} \forall A \in \mathcal{A}$ for some $w \geq 1$. Then for every $\beta>1$ and $a \in[0, w)$ there exists a constant $C_{4}$ so that

$$
\sum_{B \in \mathcal{B}, C \in \mathcal{C}} \mu(B \cap C)\left|\log \left(1+\frac{\rho(B, C)}{\mu(B) \mu(C)}\right)\right|^{a} \leq C_{4}\left(\psi(\Delta)(m+n)^{(1+a) \beta}+(m+n)^{a \beta-w(\beta-1)}\right)
$$

for $\Delta<\min (n, m)$ and for all $n=1,2, \ldots .\left(\right.$ As before $\mathcal{B}=\mathcal{A}^{m}, \mathcal{C}=T^{-\Delta-m} \mathcal{A}^{n}$.)

Proof. Let $m, n$ and $\Delta$ be as in the statement and put

$$
\mathcal{L}_{\ell}=\left\{(B, C) \in \mathcal{B} \times \mathcal{C}: 2^{\ell-1}<1+\frac{\rho(B, C)}{\mu(B) \mu(C)} \leq 2^{\ell}\right\}
$$

$\ell \in \mathbf{Z}$. Using the strong mixing property we obtain

$$
\sum_{B \in \mathcal{B}, C \in \mathcal{C}} \mu(B \cap C)\left|\log \left(1+\frac{\rho(B, C)}{\mu(B) \mu(C)}\right)\right|^{a}=\sum_{\ell=-\infty}^{\infty} L_{\ell}(|\ell|+\mathcal{O}(1))^{a}
$$

where $L_{\ell}=\sum_{(B, C) \in \mathcal{L}_{\ell}} \mu(B \cap C)$. Since $\rho(B, C)=\mathcal{O}(1)\left(2^{\ell}-1\right) \mu(B) \mu(C)$ we get $\mathcal{O}(\psi(\Delta))=\sum_{(B, C) \in \mathcal{L}_{\ell}} \rho(B, C)=$ $\mathcal{O}(1)\left(2^{\ell}-1\right) L_{\ell}^{\times}$where $L_{\ell}^{\times}=\sum_{(B, C) \in \mathcal{L}_{\ell}} \mu(B) \mu(C)$. Hence for $\ell>0$ one obtains $L_{\ell}^{\times}=\mathcal{O}(\psi(\Delta)) 2^{-\ell}$ and

if $\ell<0$ then $L_{\ell}^{\times}=\mathcal{O}(\psi(\Delta))$. Also note that if $\ell=0$ then $\left|\log \left(1+\frac{\rho(B, C)}{\mu(B) \mu(C)}\right)\right|=\mathcal{O}\left(\frac{\rho(B, C)}{\mu(B) \mu(C)}\right)$ and

$$
\sum_{(B, C) \in \mathcal{L}_{0}} \mu(B \cap C)\left|\log \left(1+\frac{\rho(B, C)}{\mu(B) \mu(C)}\right)\right|^{a}=\mathcal{O}(1) \sum_{(B, C) \in \mathcal{L}_{0}} \rho(B, C)=\mathcal{O}(\psi(\Delta)) .
$$

We separately estimate (i) for $\ell \geq 1$ and (ii) for $\ell \leq-1$ :

(i) Since $\mu(B \cap C)=\left(1+\frac{\rho(B, C)}{\mu(B) \mu(C)}\right) \mu(B) \mu(C)$ we get for $\ell \geq 1$ :

$$
2^{\ell-1} L_{\ell}^{\times}=\sum_{(B, C) \in \mathcal{L}_{\ell}} \mu(B) \mu(C) 2^{\ell-1} \leq L_{\ell} \leq \sum_{(B, C) \in \mathcal{L}_{\ell}} \mu(B) \mu(C) 2^{\ell}=2^{\ell} L_{\ell}^{\times}
$$

Thus

$$
\sum_{\ell=1}^{(m+n)^{\beta}} \ell^{a} L_{\ell} \leq \sum_{\ell=1}^{(m+n)^{\beta}} \ell^{a} 2^{\ell} L_{\ell}^{\times} \leq \sum_{\ell=1}^{(m+n)^{\beta}} \ell^{a} \frac{2^{\ell}}{2^{\ell}-1} \psi(\Delta) \leq c_{1} \psi(\Delta)(m+n)^{(1+a) \beta} .
$$


For $\ell>(m+n)^{\beta}$ we use that $\mu(B \cap C) \geq 2^{\ell-1} \mu(B) \mu(C)$ on $\mathcal{L}_{\ell}$ which implies $\mu(B) \mu(C) \leq 2^{1-\ell}$ and $\mu(B \cap C) \leq \min (\mu(B), \mu(C)) \leq 2^{-\frac{\ell-1}{2}}$. Hence, on $\mathcal{L}_{\ell}$ one has $|\log \mu(B \cap C)| \geq(\ell-1) \log \sqrt{2}$. Similarly to the previous lemma put

$$
D_{k}=\bigcup_{(B, C) \in \mathcal{B} \times \mathcal{C}, k-1<|\log \mu(B \cap C)| \leq k}(B \cap C)
$$

and use Corollary 9 to get $\left(\right.$ as $\left.K_{w}\left(\mathcal{A}^{n+m+\Delta}\right) \geq K_{w}(\mathcal{B} \vee \mathcal{C})\right)$

$$
C_{2}(w)(n+m+\Delta)^{w} \geq K_{w}(\mathcal{B} \vee \mathcal{C}) \geq \sum_{k=1}^{\infty} \mu\left(D_{k}\right)(k-1)^{w} \geq c_{2}(n+m)^{\beta(w-a)} \sum_{k=\left[(n+m)^{\beta}\right]+1}^{\infty} \mu\left(D_{k}\right) k^{a}
$$

We thus obtain (using that $\Delta<\min (n, m)$ )

$$
\begin{aligned}
\sum_{\ell=(n+m)^{\beta}}^{\infty} \ell^{a} L_{\ell} & \leq \frac{1}{\log \sqrt{2}} \sum_{(B, C) \in \mathcal{B} \times \mathcal{C},|\log \mu(B \cap C)| \geq(n+m)^{\beta}}|\log \mu(B \cap C)|^{a} \mu(B \cap C) \\
& \leq \frac{1}{\log \sqrt{2}} \sum_{k=\left[(n+m)^{\beta}\right]+1}^{\infty} k^{a} \mu\left(D_{k}\right) \\
& \leq c_{3} \frac{(n+m)^{a \beta}}{(n+m)^{(\beta-1) w}}
\end{aligned}
$$

for some $c_{3}$ (which depends on $w$ ).

(ii) For negative values of $\ell$ we use $L_{\ell} \leq 2^{\ell} L_{\ell}^{\times} \leq c_{4} 2^{\ell} \psi(\Delta)$ which gives

$$
\sum_{\ell=-\infty}^{0}|\ell|^{a} L_{\ell} \leq c_{4} \sum_{\ell=0}^{\infty} \ell^{a} 2^{-\ell} \psi(\Delta) \leq c_{5} \psi(\Delta)
$$

Combining (i) and (ii) yields

$$
\sum_{\ell=-\infty}^{\infty} L_{\ell}(|\ell|+\mathcal{O}(1))^{a} \leq\left(c_{1}+c_{4}\right) \psi(\Delta)(m+n)^{(1+a) \beta}+c_{3}(m+n)^{a \beta-w(\beta-1)}
$$

which concludes the proof.

\subsection{Entropy}

The main purpose of this section is to obtain rates of convergence for the entropy (Lemma 13).

Lemma 12 Under the assumptions of Lemma 11 for every $\beta>1$ there exists a constant $C_{5}$ so that for all $n$ :

$$
|H(\mathcal{B} \vee \mathcal{C})-(H(\mathcal{B})+H(\mathcal{C}))| \leq C_{5}\left(\psi(\Delta) n^{2 \beta}+n^{\beta-(\beta-1) w}\right)
$$

where $\mathcal{B}=\mathcal{A}^{n}, \mathcal{C}=T^{-\Delta-n} \mathcal{A}^{n}$.

Proof. Using the uniform strong mixing property $\mu(B \cap C)=\mu(B) \mu(C)+\rho(B, C)$ we obtain

$$
\begin{aligned}
H(\mathcal{B} \vee \mathcal{C}) & =\sum_{B \in \mathcal{B}, C \in \mathcal{C}} \mu(B \cap C) \log \frac{1}{\mu(B \cap C)} \\
& =\sum_{B, C} \mu(B \cap C)\left(\log \frac{1}{\mu(B)}+\log \frac{1}{\mu(C)}-\log \left(1+\frac{\rho(B, C)}{\mu(B) \mu(C)}\right)\right) \\
& =H(\mathcal{B})+H(\mathcal{C})+E,
\end{aligned}
$$


where by Lemma 11 (with $a=1$ )

$$
E=-\sum_{B \in \mathcal{B}, C \in \mathcal{C}} \mu(B \cap C) \log \left(1+\frac{\rho(B, C)}{\mu(B) \mu(C)}\right)=\mathcal{O}\left(\psi(\Delta) n^{2 \beta}+n^{\beta-(\beta-1) w}\right) .
$$

This proves the lemma.

Lemma 13 Under the assumptions of Lemma 11 there exists a constant $C_{6}$ so that $\left(H_{m}=H\left(\mathcal{A}^{m}\right)\right)$

$$
\left|\frac{H_{m}}{m}-h\right| \leq C_{6} \frac{1}{m^{\gamma}}
$$

for all $m$, where $\gamma \in\left(0,1-\frac{2 w}{p(w-1)}\right)$ if $\psi$ decays polynomially with power $p>\frac{2 w}{w-1}$ and $\gamma \in(0,1)$ if $\psi$ decays faster than polynomially.

Proof. Let $m$ be an integer. Let $\mathcal{B}=\mathcal{A}^{u-\Delta}, \mathcal{C}=T^{-u} \mathcal{A}^{u-\Delta}$ and $\mathcal{D}=T^{-u} \mathcal{A}^{2 \Delta}$, then by Lemma 12,

$$
H_{2 u}=2 H_{u-\Delta}+\mathcal{O}\left(H_{2 \Delta}\right)+\mathcal{O}\left(\psi(\Delta) u^{2 \beta}+n^{\beta-(\beta-1) w}\right) .
$$

If we choose $\delta \in\left(\frac{2 w}{p(w-1)}, 1\right)$ and put $\beta=\frac{w}{w-1}$ then $\Delta=\mathcal{O}\left(u^{\delta}\right)$ implies that $\psi(\Delta) u^{2 \beta}+u^{\beta-(\beta-1) w}=\mathcal{O}(1)$. With $\Delta=\left[u^{\delta}\right]$ we thus obtain $H_{2 u}=2 H_{u}+\mathcal{O}(\Delta)=2 H_{u}+\mathcal{O}\left(u^{\delta}\right)$ as $H_{2 \Delta}=\mathcal{O}(\Delta)$ and $H_{u-\Delta}=H_{u}+\mathcal{O}(\Delta)$. Iterating this estimate yields the following bound along exponential progression:

$$
H_{2^{i} m}=2^{i} H_{m}+\sum_{j=0}^{i-1} 2^{i-1-j} \mathcal{O}\left(\left(2^{j} m\right)^{\delta}\right)=2^{i} H_{m}+\mathcal{O}\left(m^{\delta} 2^{i}\right) .
$$

To get bounds for arbitrary (large) integers $n$ we do the following dyadic argument: Let $n=k m+r$ where $0 \leq r<m$ and consider the binary expansion of: $k=\sum_{i=0}^{\ell} \epsilon_{i} 2^{i}$, where $\epsilon_{i}=0,1\left(\epsilon_{\ell}=1, \ell=\left[\log _{2} k\right]\right)$. We also put $k_{j}=\sum_{i=0}^{j} \epsilon_{i} 2^{i}\left(k_{\ell}=k\right)$. Obviously $k_{j}=k_{j-1}+\epsilon_{j} 2^{j} \leq 2^{j+1}$. If $\epsilon_{j}=1$ then we separate the 'first' block of length $k_{j-1} m$ from the 'second' block of length $2^{j} m$ by a gap of length $2\left[\left(k_{j-1} m\right)^{\delta}\right]$ which we cut away in equal parts from the two adjacent blocks). We thus obtain $\left(H_{0}=0\right)$

$$
H_{m k_{j}}=H_{\epsilon_{j} 2^{j} m+k_{j-1} m}=H_{\epsilon_{j} 2^{j} m}+H_{k_{j-1} m}+\mathcal{O}\left(\epsilon_{j}\left(k_{j-1} m\right)^{\delta}\right)=H_{\epsilon_{j} 2^{j} m}+H_{k_{j-1} m}+\mathcal{O}\left(\epsilon_{j}\left(2^{j} m\right)^{\delta}\right)
$$

for $j=0,1, \ldots, \ell-1$. Iterating this formula and summing over $j$ yields

$$
H_{k m}=\sum_{j=0}^{\ell} \epsilon_{j}\left(2^{j} H_{m}+\mathcal{O}\left(m^{\delta} 2^{j}\right)\right)=k H_{m}+\mathcal{O}\left(m^{\delta} 2^{\ell}\right) .
$$

The contribution made by the remainder of length $r$ is easily bounded by

$$
\left|H_{n}-H_{k m}\right| \leq \sigma\left(\mathcal{A}^{n} \mid \mathcal{A}^{k m}\right) \leq c_{1} r \leq c_{1} m
$$

Consequently

$$
H_{n}=k H_{m}+\mathcal{O}\left(m^{\delta} 2^{\ell}\right)+\mathcal{O}(m)=k H_{m}+\mathcal{O}\left(m^{\delta} k\right)
$$

as $2^{\ell} \leq k \leq 2^{\ell+1}$. Dividing by $n$ and letting $n$ go to infinity $(k \rightarrow \infty)$ yields

$$
h=\liminf _{n \rightarrow \infty} \frac{H_{n}}{n}=\frac{H_{m}}{m}+\mathcal{O}\left(m^{\delta-1}\right)
$$

for all $m$ large enough. 


\subsection{The variance}

In this section we prove part (I) of Theorem 2 and moreover obtain convergence rates which will be needed to prove part (II) in section 4.

Proposition 14 Let $\mu$ be uniformly strong mixing and assume that $K_{w}(\mathcal{A})<\infty$ and $\mu(A) \leq e^{-w} \forall A \in$ $\mathcal{A}$ for some $w>2$. Assume that $\psi$ is at least polynomially decaying with power $p>6+\frac{8}{w-2}$. Then the limit

$$
\sigma^{2}=\lim _{n \rightarrow \infty} \frac{1}{n} \sigma^{2}\left(\mathcal{A}^{n}\right)
$$

exists and is finite. Moreover for every $\eta<\eta_{0}=2 \frac{(p-2)(w-2)}{(w-2)(p+2)+8}$ there exists a constant $C_{7}$ so that for all $n \in \mathbb{N}:$

$$
\left|\sigma^{2}-\frac{\sigma^{2}\left(\mathcal{A}^{n}\right)}{n}\right| \leq \frac{C_{7}}{n^{\eta}} .
$$

Moreover, if the partition $\mathcal{A}$ is infinite, then $\sigma$ is strictly positive.

Proof. With $\mathcal{B}=\mathcal{A}^{n}, \mathcal{C}=T^{-n-\Delta} \mathcal{A}^{n}$ we have by Lemma $12 H(\mathcal{B} \vee \mathcal{C})=H(\mathcal{B})+H(\mathcal{C})+\mathcal{O}\left(\psi(\Delta) n^{2 \beta}+n^{\beta-(\beta-1) w}\right)$, and get for the variance

$$
\begin{aligned}
\sigma^{2}(\mathcal{B} \vee \mathcal{C}) & =\sum_{B \in \mathcal{B}, C \in \mathcal{C}} \mu(B \cap C)\left(\log \frac{1}{\mu(B \cap C)}-H(\mathcal{B} \vee \mathcal{C})\right)^{2} \\
& =\sum_{B, C} \mu(B \cap C)\left(J_{\mathcal{B}}(B)+J_{\mathcal{C}}(C)+\mathcal{O}\left(\psi(\Delta) n^{2 \beta}+n^{\beta-(\beta-1) w}\right)-\log \left(1+\frac{\rho(B, C)}{\mu(B) \mu(C)}\right)\right)^{2} .
\end{aligned}
$$

By Minkowski's inequality:

$$
|\sigma(\mathcal{B} \vee \mathcal{C})-\sqrt{E(\mathcal{B}, \mathcal{C})}| \leq c_{1}\left(\psi(\Delta) n^{2 \beta}+n^{\beta-(\beta-1) w}\right)+\sqrt{F(\mathcal{B}, \mathcal{C})}
$$

$\left(c_{1}>0\right)$ where (by Lemma 11 with $a=2$ )

$$
F(\mathcal{B}, \mathcal{C})=\sum_{B \in \mathcal{B}, C \in \mathcal{C}} \mu(B \cap C) \log ^{2}\left(1+\frac{\rho(B, C)}{\mu(B) \mu(C)}\right) \leq c_{2}\left(\psi(\Delta) n^{3 \beta}+n^{2 \beta-(\beta-1) w}\right),
$$

and

$$
\begin{aligned}
E(\mathcal{B}, \mathcal{C}) & =\sum_{B \in \mathcal{B}, C \in \mathcal{C}} \mu(B \cap C)\left(J_{\mathcal{B}}(B)+J_{\mathcal{C}}(C)\right)^{2} \\
& =\sum_{B, C} \mu(B \cap C)\left(J_{\mathcal{B}}(B)^{2}+J_{\mathcal{C}}(C)^{2}\right)+2 G(\mathcal{B}, \mathcal{C}) \\
& =\sigma^{2}(\mathcal{B})+\sigma^{2}(\mathcal{C})+2 G(\mathcal{B}, \mathcal{C}) .
\end{aligned}
$$

Since $J_{\mathcal{B}}$ and $J_{\mathcal{C}}$ have average zero the remainder term

$$
\begin{aligned}
G(\mathcal{B}, \mathcal{C}) & =\sum_{B \in \mathcal{B}, C \in \mathcal{C}} \mu(B \cap C) J_{\mathcal{B}}(B) J_{\mathcal{C}}(C) \\
& =\sum_{B, C}(\mu(B) \mu(C)+\rho(B, C)) J_{\mathcal{B}}(B) J_{\mathcal{C}}(C) \\
& =\sum_{B, C} \rho(B, C) J_{\mathcal{B}}(B) J_{\mathcal{C}}(C)
\end{aligned}
$$


which is estimated using Schwarz' inequality as follows

$$
|G(\mathcal{B}, \mathcal{C})| \leq \sum_{B, C}|\rho(B, C)| \cdot\left|J_{\mathcal{B}}(B)\right| \cdot\left|J_{\mathcal{C}}(C)\right| \leq \psi(\Delta) \sigma(\mathcal{B}) \sigma(\mathcal{C}) .
$$

Hence

$$
\sigma(\mathcal{B} \vee \mathcal{C}) \leq \sqrt{\sigma^{2}(\mathcal{C})+\sigma^{2}(\mathcal{B})+\psi(\Delta) \sigma(\mathcal{B}) \sigma(\mathcal{C})}+c_{4} \sqrt{\psi(\Delta) n^{3 \beta}+n^{2 \beta-(\beta-1) w}}
$$

Next we fill the gap of length $\Delta$ for which we use Lemma 10 and Corollary 9

$$
\left|\sigma\left(\mathcal{A}^{2 n+\Delta}\right)-\sigma(\mathcal{B} \vee \mathcal{C})\right| \leq \sigma\left(T^{-n} \mathcal{A}^{\Delta} \mid \mathcal{B} \vee \mathcal{C}\right) \leq \sqrt{K_{2}\left(T^{-n} \mathcal{A}^{\Delta}\right)}=\sqrt{K_{2}\left(\mathcal{A}^{\Delta}\right)} \leq c_{5} \Delta
$$

Since by assumption $\psi(\Delta) \leq c_{6} \Delta^{-p}$ for some $p>6+\frac{8}{w-2}$ we take can $\delta=\frac{4 w}{(p+2)(w-2)+8}$ and $\beta=\frac{2+p}{4} \delta$ (in particular $\delta<\frac{1}{2}$ ). Then, with $\Delta=\left[n^{\delta}\right]$ we get $\psi(\Delta) n^{4 \beta}+n^{2 \beta-(\beta-1) w} \leq \Delta^{2}$. Therefore, as $\sigma(\mathcal{B})=$ $\sigma(\mathcal{C})=\sigma_{n}\left(\right.$ where $\left.\sigma_{n}=\sigma\left(\mathcal{A}^{n}\right)\right)$, one has

$$
\sigma_{2 n+\left[n^{\delta}\right]} \leq \sqrt{(2+\psi(\Delta)) \sigma_{n}^{2}+c_{7} n^{2 \delta}} \leq \sqrt{2 \sigma_{n}^{2}+c_{7} n^{2 \delta}}
$$

where in the last step we took advantage of the a priori estimates from Corollary $9 \sigma^{2}\left(\mathcal{A}^{n}\right) \leq K_{2}\left(\mathcal{A}^{n}\right) \leq$ $C_{2} n^{2}$ and the choice of $\delta$ which implies that $\psi(\Delta) n^{2}=\mathcal{O}(1)$. Since $2 \delta<1$ one has $\sigma_{k}^{2} \leq c_{8} k$ for all $k$ and some constant $c_{8}$. Given $n_{0}$ let us put recursively $n_{j+1}=2 n_{j}+\left[n_{j}^{\delta}\right](j=0,1,2, \ldots)$. Then $2^{j} n_{0} \leq n_{j} \leq 2^{j} n_{0} \prod_{i=0}^{j-1}\left(1+\frac{1}{2} n_{i}^{\delta-1}\right)$ where the product is bounded by

$$
\prod_{i=0}^{j-1}\left(1+\frac{1}{2} n_{i}^{\delta-1}\right) \leq \prod_{i=0}^{j-1}\left(1+\frac{1}{n_{0}^{1-\delta} 2^{(1-\delta) i+1}}\right) \leq \exp \frac{c_{9}}{n_{0}^{1-\delta}}
$$

In the same fashion one shows that $\left|\sigma_{n_{j+1}}^{2}-2 \sigma_{n_{j}}^{2}\right| \leq c_{7} n_{j}^{2 \delta}$ implies

$$
2^{j} \sigma_{n_{0}}^{2} \exp -\frac{c_{10}}{n_{0}^{1-2 \delta}} \leq \sigma_{n_{j}}^{2} \leq 2^{j} \sigma_{n_{0}}^{2} \exp \frac{c_{10}}{n_{0}^{1-2 \delta}}
$$

Hence

which simplifies to

$$
\frac{2^{j} \sigma_{n_{0}}^{2}}{2^{j} n_{0}} \exp -\left(\frac{c_{10}}{n_{0}^{1-2 \delta}}+\frac{c_{9}}{n_{0}^{1-\delta}}\right) \leq \frac{\sigma_{n_{j}}^{2}}{n_{j}} \leq \frac{2^{j} \sigma_{n_{0}}^{2}}{2^{j} n_{0}} \exp \frac{c_{10}}{n_{0}^{1-2 \delta}}
$$

$$
\frac{\sigma_{n_{j}}^{2}}{n_{j}}=\frac{\sigma_{n_{0}}^{2}}{n_{0}}\left(1+\mathcal{O}\left(\frac{1}{n_{0}^{1-2 \delta}}\right)\right)=\frac{\sigma_{n_{0}}^{2}}{n_{0}}+\mathcal{O}\left(\frac{1}{n_{0}^{2-2 \delta}}\right) .
$$

As $w>2$ one has $\sigma_{n_{0}}<\infty$. Taking limsup as $j \rightarrow \infty$ and $n_{0} \rightarrow \infty$ shows that the $\operatorname{limit}^{2}=\lim _{n} \frac{\sigma_{n}^{2}}{n}$ exists and satisfies moreover $\left|\sigma^{2}-\frac{\sigma_{n}^{2}}{n}\right| \leq C_{7} n^{-(2-2 \delta)}$ for some $C_{7}$. Now we obtain the statement in the proposition for all $\eta<2-2 \delta=2 \frac{(p-2)(w-2)}{(p+2)(w-2)+8}$.

In order to prove the last statement of the proposition let $\mathcal{A}$ be an infinite partition. If we choose $n_{0}$ large enough so that the error term $\mathcal{O}\left(n_{0}^{-(1-2 \delta)}\right)$ in equation (2) is $<\frac{1}{2}$, then $\sigma_{n_{j}}^{2}>\frac{1}{2} n_{j} \sigma_{n_{0}}^{2}$ for all $j$. Since

$$
\begin{aligned}
\sigma_{n_{0}}^{2} & =\sum_{A \in \mathcal{A}^{n_{0}}} \mu(A) \log ^{2} \mu(A)-\sum_{A, B \in \mathcal{A}^{n_{0}}} \mu(A) \mu(B) \log \mu(A) \log \mu(B) \\
& =\frac{1}{2} \sum_{A, B} \mu(A) \mu(B)\left(\log ^{2} \mu(A)+\log ^{2} \mu(B)\right)-\sum_{A, B} \mu(A) \mu(B) \log \mu(A) \log \mu(B) \\
& =\frac{1}{2} \sum_{A, B \in \mathcal{A}^{n_{0}}} \mu(A) \mu(B) \log ^{2} \frac{\mu(A)}{\mu(B)}
\end{aligned}
$$


we conclude that $\sigma_{n_{0}}^{2}>0$. Hence $\sigma^{2}=\lim _{n} \frac{\sigma_{n}^{2}}{n}$ is strictly positive.

Remarks: (i) It is well known that for finite partitions the measure has variance zero if it is a Gibbs state for a potential which is a coboundary.

(ii) This proposition implies in particular that the $\operatorname{limit}_{n \rightarrow \infty} \frac{1}{n^{2}} K_{2}\left(\mathcal{A}^{n}\right)$ exists and is equal to $h^{2}$.

(iii) An application of Chebycheff's inequality gives the large deviation type estimate $\left(\sigma_{n}=\sigma\left(J_{n}\right)\right)$

$$
\mathbb{P}\left(\frac{1}{n} J_{n}(x) \geq t\right) \leq \frac{\sigma_{n}^{2}}{n^{2} t^{2}}=\mathcal{O}\left(\frac{1}{n t^{2}}\right)
$$

\subsection{Higher order moments}

In the proof of Theorem 2 part (II) we will need estimates on the third and fourth moments of $J_{n}$. We first estimate the fourth moment and then use Hölder's inequality to bound the third moment. Denote by

$$
M_{w}(\mathcal{B})=\sum_{B \in \mathcal{B}} \mu(B)\left|J_{\mathcal{B}}(B)\right|^{w} .
$$

the $w$ th (absolute) moment of the function $J_{\mathcal{B}}$. By Minkowski's inequality

$$
M_{4}^{\frac{1}{4}}(\mathcal{B} \vee \mathcal{C})=\sqrt[4]{\mu\left(J_{\mathcal{C} \mid \mathcal{B}}+J_{\mathcal{B}}\right)^{4}} \leq \sqrt[4]{\mu\left(J_{\mathcal{C} \mid \mathcal{B}}^{4}\right)}+\sqrt[4]{\mu\left(J_{\mathcal{B}}^{4}\right)}=M_{4}^{\frac{1}{4}}(\mathcal{C} \mid \mathcal{B})+M_{4}^{\frac{1}{4}}(\mathcal{B})
$$

where $M_{w}(\mathcal{C} \mid \mathcal{B})=\sum_{B \in \mathcal{B}, C \in \mathcal{C}} \mu(B \cap C)\left|J_{\mathcal{C} \mid \mathcal{B}}(B \cap C)\right|^{w}$ are the conditional moments. It follows from Corollary 9 that the absolute moments for the joins $\mathcal{A}^{n}$ can roughly be bounded by $M_{w}\left(\mathcal{A}^{n}\right) \leq K_{w}\left(\mathcal{A}^{n}\right) \leq$ $C_{2} n^{w}$. This estimate however is useless to prove Theorem 2 and the purpose of the next proposition is to reduce the exponent $w$ to $\frac{1}{2} w$ in the cases $w=3,4$. One can of course get these improved estimates also for $w$ larger than 4 (as long as $K_{w}(\mathcal{A})<\infty$ ) but we don't need those higher order moments here.

Proposition 15 Let $\mu$ be uniformly strong mixing and assume that $K_{w}(\mathcal{A})<\infty$ and $\mu(A) \leq e^{-w} \forall A \in$ $\mathcal{A}$ for some $w>4$. Also assume that $\psi$ decays at least polynomially with power $>8+\frac{24}{w-4}$. Then there exists a constant $C_{8}$ so that for all $n$

$$
M_{4}\left(\mathcal{A}^{n}\right) \leq C_{8} n^{2}
$$

Proof. With $\mathcal{B}=\mathcal{A}^{n}, \mathcal{C}=T^{-\Delta-n} \mathcal{A}^{n}$ we get (by Lemma 12) $H(\mathcal{B} \vee \mathcal{C})=H(\mathcal{B})+H(\mathcal{C})+\mathcal{O}\left(\psi(\Delta) n^{2 \beta}+\right.$ $\left.n^{1-(\beta-1) w}\right)$ and with Minkowsky's inequality (on $L^{4}$ spaces)

$$
\begin{aligned}
M_{4}^{\frac{1}{4}}(\mathcal{B} \vee \mathcal{C}) & =\left(\sum_{B \in \mathcal{B}, C \in \mathcal{C}} \mu(B \cap C)\left(\log \frac{1}{\mu(B \cap C)}-H(\mathcal{B} \vee \mathcal{C})\right)^{4}\right)^{\frac{1}{4}} \\
& \leq E_{4}^{\frac{1}{4}}(\mathcal{B}, \mathcal{C})+\mathcal{O}\left(\psi(\Delta) n^{2 \beta}+n^{\beta-(\beta-1) w}\right)+F_{4}^{\frac{1}{4}}(\mathcal{B}, \mathcal{C})
\end{aligned}
$$

where by Lemma 11 (with $a=4$ )

$$
F_{4}(\mathcal{B}, \mathcal{C})=\sum_{B \in \mathcal{B}, C \in \mathcal{C}} \mu(B \cap C) \log ^{4}\left(1+\frac{\rho(B, C)}{\mu(B) \mu(C)}\right)=\mathcal{O}\left(\psi(\Delta) n^{5 \beta}+n^{4 \beta-(\beta-1) w}\right)
$$

and

$$
\begin{aligned}
E_{4}(\mathcal{B}, \mathcal{C}) & =\sum_{B \in \mathcal{B}, C \in \mathcal{C}} \mu(B \cap C)\left(J_{\mathcal{B}}(B)+J_{\mathcal{C}}(C)\right)^{4} \\
& =M_{4}(\mathcal{B})+M_{4}(\mathcal{C})+\sum_{B, C} \mu(B \cap C)\left(4 J_{\mathcal{B}}(B)^{3} J_{\mathcal{C}}(C)+6 J_{\mathcal{B}}(B)^{2} J_{\mathcal{C}}(C)^{2}+4 J_{\mathcal{B}}(B) J_{\mathcal{C}}(C)^{3}\right)
\end{aligned}
$$


We look individually at the terms in the bracket:

$$
\begin{aligned}
\left|\sum_{B \in \mathcal{B}, C \in \mathcal{C}} \mu(B \cap C) J_{\mathcal{B}}(B)^{3} J_{\mathcal{C}}(C)\right| & =\left|\sum_{B, C}(\mu(B) \mu(C)+\rho(B, C)) J_{\mathcal{B}}(B)^{3} J_{\mathcal{C}}(C)\right| \\
& \leq \sum_{B, C}|\rho(B, C)| \cdot\left|J_{\mathcal{B}}(B)\right|^{3}\left|J_{\mathcal{C}}(C)\right| \\
& \leq \psi(\Delta) M_{3}(\mathcal{B}) \sigma(\mathcal{C}) .
\end{aligned}
$$

because $J_{\mathcal{B}}$ and $J_{\mathcal{C}}$ have zero average Schwarz inequality. In the same way we get

$$
\left|\sum_{B \in \mathcal{B}, C \in \mathcal{C}} \mu(B \cap C) J_{\mathcal{B}}(B) J_{\mathcal{C}}(C)^{3}\right| \leq \psi(\Delta) \sigma(\mathcal{B}) M_{3}(\mathcal{C}) .
$$

Moreover

$$
\begin{aligned}
\sum_{B \in \mathcal{B}, C \in \mathcal{C}} \mu(B \cap C) J_{\mathcal{B}}(B)^{2} J_{\mathcal{C}}(C)^{2} & =\sum_{B, C}(\mu(B) \mu(C)+\rho(B, C)) J_{\mathcal{B}}(B)^{2} J_{\mathcal{C}}(C)^{2} \\
& =\sigma^{2}(\mathcal{B}) \sigma^{2}(\mathcal{C})+G(\mathcal{B}, \mathcal{C})
\end{aligned}
$$

where

$$
|G(\mathcal{B}, \mathcal{C})|=\left|\sum_{B \in \mathcal{B}, C \in \mathcal{C}} \rho(B, C) J_{\mathcal{B}}(B)^{2} J_{\mathcal{C}}(C)^{2}\right| \leq \psi(\Delta) \sigma^{2}(\mathcal{B}) \sigma^{2}(\mathcal{C})
$$

Thus

$$
E_{4}(\mathcal{B}, \mathcal{C})=M_{4}(\mathcal{B})+M_{4}(\mathcal{C})+(6+\psi(\Delta)) \sigma^{2}(\mathcal{B}) \sigma^{2}(\mathcal{C})+\psi(\Delta)\left(M_{3}(\mathcal{B}) \sigma(\mathcal{C})+\sigma(\mathcal{B}) M_{3}(\mathcal{C})\right)
$$

As $\sigma^{2}(\mathcal{B})=\sigma^{2}(\mathcal{C})=\sigma_{n}^{2} \leq c_{1} n$ (Proposition 14) and since by assumption $\psi(\Delta)=\mathcal{O}\left(\Delta^{-p}\right.$ ) where $p>8+\frac{24}{w-4}$ we can choose $\beta=1+\frac{2}{w-4}, \delta=\frac{1}{p}\left(4+\frac{12}{w-4}\right)$ and put $\Delta=\left[n^{\delta}\right]$. This implies $\Delta<\sqrt{n}$ (as $\left.\delta<\frac{1}{2}\right)$ and $\psi(\Delta) n^{6 \beta}+n^{4 \beta-(\beta-1) w}=\mathcal{O}\left(n^{2}\right)$. Using the a priori estimates $M_{3}\left(\mathcal{A}^{n}\right) \leq K_{3}\left(\mathcal{A}^{n}\right) \leq C_{2} n^{3}$ we obtain in particular that $\psi(\Delta)\left(M_{3}(\mathcal{B}) \sigma(\mathcal{C})+\sigma(\mathcal{B}) M_{3}(\mathcal{C})\right)=\mathcal{O}\left(n^{2}\right)$ and therefore

$$
\left.M_{4}^{\frac{1}{4}}(\mathcal{B} \vee \mathcal{C})=\sqrt[4]{M_{4}(\mathcal{C})+M_{4}(\mathcal{B})+c_{2} n^{2}}+\mathcal{O}(\psi(\Delta)) n^{2 \beta}+n^{\beta-(\beta-1) w}\right)
$$

where the error term on the right hand side is $\mathcal{O}\left(n^{-3}\right)$. To fill in the gap of length $\Delta$ we use Lemma 10 and the estimate on $K_{4}$ (Corollary 9):

$$
\left|M_{4}^{\frac{1}{4}}\left(\mathcal{A}^{2 n+\Delta}\right)-M_{4}^{\frac{1}{4}}(\mathcal{B} \vee \mathcal{C})\right| \leq M_{4}^{\frac{1}{4}}\left(\mathcal{A}^{2 n+\Delta} \mid \mathcal{B} \vee \mathcal{C}\right) \leq K_{4}^{\frac{1}{4}}\left(\mathcal{A}^{\Delta}\right) \leq c_{3} \Delta
$$

Hence

$$
M_{4}^{\frac{1}{4}}\left(\mathcal{A}^{n^{\prime}}\right) \leq \sqrt[4]{2 M_{4}\left(\mathcal{A}^{n}\right)+c_{2} n^{2}}+c_{3} \Delta \leq \sqrt[4]{2 M_{4}\left(\mathcal{A}^{n}\right)+c_{4} n^{2}}
$$

(as $\Delta \leq \sqrt{n}$ ), and by induction $M_{4}\left(\mathcal{A}^{k}\right) \leq C_{8} k^{2}$ (with $C_{8} \geq c_{4} / 2$ ).

A Hölder estimate lets us now estimate the third absolute moments of $J_{n}$ as follows.

Corollary 16 Under the assumptions of Proposition 15 there exists a constant $C_{9}$ so that for all $n$

$$
M_{3}\left(\mathcal{A}^{n}\right) \leq C_{9} n^{\frac{3}{2}} .
$$




\section{Proof of Theorem 2 (CLT for Shannon-McMillan-Breiman)}

As before $N(t)$ denotes the normal distribution with zero mean and variance one. We will first show the following result (in which $n h$ has been replaced by $H_{n}$ and $\sigma \sqrt{n}$ by $\sigma_{n}$ ).

Theorem 17 Under the assumptions of Theorem 0 one has:

(I) The limit $\sigma^{2}=\lim _{n \rightarrow \infty} \frac{1}{n}\left(K_{2}\left(\mathcal{A}^{n}\right)-H_{n}^{2}\right)$ exists (and is positive if $|\mathcal{A}|=\infty$ ).

(II) If $\sigma>0$ then

$$
\mathbb{P}\left(\frac{I_{n}-H_{n}}{\sigma_{n}} \leq t\right)=N(t)+\mathcal{O}\left(\frac{1}{n^{\kappa}}\right)
$$

for all $t$ and all

(i) $\kappa<\frac{1}{10}-\frac{3}{5} \frac{w}{(p+2)(w-2)+6}$ if $\psi$ decays polynomially with power $p$,

(ii) $\kappa<\frac{1}{10}$ if $\psi$ decays hyper polynomially.

Proof of Theorem 17. It is enough to prove the theorem with the partition $\mathcal{A}$ replaced by one of its joins $\mathcal{A}^{k}$ for some $k$. Since by Lemma $7 \mu(A) \leq e^{-w} \forall A \in \mathcal{A}^{k}$ for some $k \geq 1$ we therefore replace the original partition by $\mathcal{A}^{k}$ and will henceforth assume that $\mu(A) \leq e^{-w}$ for all $A \in \mathcal{A}$.

Theorem 17 part (I) follows from Proposition 14. For the proof of part (II) let us assume that $\sigma$ is positive. We will use Stein's method to prove the CLT in the form of the following proposition which is modelled after 37:

Proposition 18 [33] Let $\left(W, W^{\prime}\right)$ be an exchangeable pair so that $\mathbb{E}(W)=0$ and $\operatorname{var}(W)=1$ and assume

$$
\mathbb{E}\left(W^{\prime} \mid W\right)=(1-\lambda) W
$$

for some $\lambda \in(0,1)$. Then for all real $t$ :

$$
|\mathbb{P}(W \leq t)-N(t)| \leq \frac{6}{\lambda} \sqrt{\operatorname{var}\left(\mathbb{E}\left(\left(W^{\prime}-W\right)^{2} \mid W\right)\right)}+6 \sqrt{\frac{1}{\lambda} \mathbb{E}\left(\left|W^{\prime}-W\right|^{3}\right)} .
$$

We proceed in five steps: (A) We begin with a classical 'big block-small block' argument and approximate $W_{n}=\frac{J_{n}}{\sigma_{n}}$ by a sum of random variables which are separated by gaps. In (B) we then replace those random variables by independent random variables. In (C) we define the interchangeable pair in the usual way and estimate the terms on the right hand side of Proposition 18, In (D) and (E) we estimate the effects the steps $(\mathrm{A})$ and $(\mathrm{B})$ have on the distributions.

We approximate $W_{n}=\frac{J_{n}}{\sigma_{n}}$ (clearly $\mathbb{E}\left(W_{n}\right)=0, \sigma\left(W_{n}\right)=1$ ) by the random variable $\hat{W}_{n}=\frac{1}{\sqrt{r}} \sum_{j=0}^{r-1} W_{m}$ 。 $T^{m^{\prime} j}$ (that is $\hat{W}_{n}=\frac{1}{\sqrt{r} \sigma_{m}} \sum_{j=0}^{r-1} J_{m} \circ T^{m^{\prime} j}$ ) where $m^{\prime}=m+\Delta$ and $n=r m+(r-1) \Delta$. (For other values of $n$ not of this form we get an additional error term of the order $m^{\prime}$.)

(A) If we put $\hat{\mathcal{A}}^{n}=\bigvee_{j=0}^{r-1} T^{-m^{\prime} j} \mathcal{A}^{m}$ then

$$
\begin{aligned}
\left\|\hat{W}_{n}-W_{n}\right\|_{2} \leq & \frac{1}{\sigma_{n}}\left\|J_{\mathcal{A}^{n}}-J_{\hat{\mathcal{A}}^{n}}\right\|_{2}+\frac{1}{\sigma_{n}}\left\|I_{\hat{\mathcal{A}}^{n}}-\sum_{j=0}^{r-1} I_{m} \circ T^{m^{\prime} j}\right\|_{2} \\
& +\frac{1}{\sigma_{n}}\left|H\left(\hat{\mathcal{A}}^{n}\right)-r H_{m}\right|+\left|\frac{1}{\sigma_{n}}-\frac{1}{\sqrt{r} \sigma_{m}}\right| \cdot\left\|\sum_{j=0}^{r-1} J_{m} \circ T^{m^{\prime} j}\right\|_{2} .
\end{aligned}
$$

We individually estimate the four terms on the right hand side as follows:

(i) By Lemma 10 and Proposition 14

$$
\left\|J_{\mathcal{A}^{n}}-J_{\hat{\mathcal{A}}^{n}}\right\|_{2}=\sigma\left(\mathcal{A}^{n} \mid \hat{\mathcal{A}}^{n}\right)=\sigma\left(\bigvee_{j=1}^{r-1} T^{-m-m^{\prime} j} \mathcal{A}^{\Delta} \mid \hat{\mathcal{A}}^{n}\right)=\sigma\left(\bigvee_{j=1}^{r-1} T^{-m-m^{\prime} j} \mathcal{A}^{\Delta}\right) \leq c_{1} r \sqrt{\Delta}
$$


(ii) If $\mathcal{D}_{k}=\bigvee_{j=0}^{k-1} T^{-m^{\prime} j} \mathcal{A}^{m}$ then $\mathcal{D}_{k+1}=\mathcal{D}_{k} \vee T^{-m^{\prime} k} \mathcal{A}^{m}, k=1,2, \ldots, r$, and by Lemma 11 $(a=2)$

$$
\begin{aligned}
\left\|I_{\mathcal{D}_{k+1}}-I_{\mathcal{D}_{k}}-I_{m} \circ T^{m^{\prime} k}\right\|_{2}^{2} & =\sum_{D \in \mathcal{D}_{k}, A \in T^{-m^{\prime} k} \mathcal{A}^{m}} \mu(D \cap A)\left(\frac{1}{\mu(D \cap A)}-\frac{1}{\mu(D)}-\frac{1}{\mu(A)}\right)^{2} \\
& =\sum_{D \in \mathcal{D}_{k}, A \in T^{-m^{\prime} k} \mathcal{A}^{m}} \mu(D \cap A) \log ^{2}\left(1+\frac{\rho(D, A)}{\mu(D) \mu(A)}\right) \\
& \leq c_{2}\left(\psi(\Delta) n^{3 \beta}+n^{2 \beta-(\beta-1) w}\right)
\end{aligned}
$$

for $k=1,2, \ldots, r$. Hence $\left(\right.$ as $\left.\mathcal{D}_{1}=\mathcal{A}^{m}\right)$

$$
\left\|I_{\hat{\mathcal{A}}^{n}}-\sum_{j=0}^{r-1} I_{m} \circ T^{m^{\prime} j}\right\|_{2} \leq \sum_{k=1}^{r}\left\|I_{\mathcal{D}_{k+1}}-I_{\mathcal{D}_{k}}-I_{m} \circ T^{m^{\prime} k}\right\|_{2} \leq c_{3} r \sqrt{\psi(\Delta) n^{3 \beta}+n^{2 \beta-(\beta-1) w}} .
$$

(iii) $\left|H\left(\hat{\mathcal{A}}^{n}\right)-r H_{m}\right| \leq c_{4} r\left(\psi(\Delta) n^{2 \beta}+n^{\beta-(\beta-1) w}\right)$ by Lemma 12 ,

(iv) Since by Proposition 14

$$
\left|\frac{1}{\sigma_{n}}-\frac{1}{\sqrt{r} \sigma_{m}}\right|=\frac{\left|\sigma_{n}-\sqrt{r} \sigma_{m}\right|}{\sqrt{r} \sigma_{n} \sigma_{m}} \leq c_{5} \frac{m^{-\eta}}{\sqrt{n}},
$$

Lemma 10 and again Proposition 14

$$
\left\|\sum_{j=0}^{r-1} J_{m} \circ T^{m^{\prime} j}\right\|_{2}=\sigma\left(\bigvee_{j=0}^{r-1} T^{-m^{\prime} j} \mathcal{A}^{m}\right) \leq r \sigma\left(\mathcal{A}^{m}\right)=\mathcal{O}(r \sqrt{m}),
$$

we obtain that the fourth term is $\mathcal{O}\left(\sqrt{r} m^{-\eta}\right)$, for any $\eta<\eta_{0}$.

Therefore, if $n$ is large enough,

$$
\begin{aligned}
\left\|\hat{W}_{n}-W_{n}\right\|_{2} & \leq c_{6}\left(\frac{r \sqrt{\Delta}}{\sqrt{n}}+\frac{r}{\sqrt{n}} \sqrt{\psi(\Delta) n^{3 \beta}+n^{2 \beta-(\beta-1) w}}+\frac{r}{\sqrt{n}}\left(\psi(\Delta) n^{2 \beta}+n^{\beta-(\beta-1) w}\right)+\frac{\sqrt{r}}{m^{\eta}}\right) \\
& \leq c_{7}\left(\frac{r \Delta}{\sqrt{n}}+r n^{\frac{3}{2} \beta-\frac{1}{2}} \sqrt{\psi(\Delta)}\left(1+n^{\frac{1}{2} \beta} \sqrt{\psi(\Delta)}\right)+r n^{\beta-\frac{1}{2}-\frac{1}{2}(\beta-1) w}+\frac{\sqrt{r}}{m^{\eta}}\right)
\end{aligned}
$$

as $\sigma_{n} \sim \sqrt{n}$ and $\beta-1>0$.

(B) Now let $X_{j}$ for $j=0,1, \ldots, r-1$ be independent random variables that have the same distributions as $W_{m} \circ T^{m^{\prime} j}, j=0,1, \ldots, r-1$. Put $D_{V_{n}}(t)$ for the distribution function of the random variable $V_{n}=\frac{1}{\sqrt{r}} \sum_{j=0}^{r-1} X_{j}$ and $D_{\hat{W}_{n}}(t)$ for the distribution function of $\hat{W}_{n}$. Since $V_{n}$ and $\hat{W}_{n}$ assume the same values, the difference between the distributions is given by (with $\mathcal{D}_{k}=\bigvee_{j=0}^{k-1} T^{-m^{\prime} j} \mathcal{A}^{m}$ as above):

$$
\begin{aligned}
\sup _{t}\left|D_{\hat{W}_{n}}(t)-D_{V_{n}}(t)\right| & \leq \sum_{A_{0} \in \mathcal{A}^{m}} \ldots \sum_{A_{r-1} \in T^{-m^{\prime}(r-1)} \mathcal{A}^{m}}\left|\mu\left(\bigcap_{j} A_{j}\right)-\prod_{j} \mu\left(A_{j}\right)\right| \\
& \leq \sum_{k=0}^{r-1} \sum_{D \in \mathcal{D}_{k}} \sum_{A \in T^{-m^{\prime} k} \mathcal{A}^{m}}|\mu(D \cap A)-\mu(D) \mu(A)| \\
& =\sum_{k=0}^{r-1} \sum_{D \in \mathcal{D}_{k}} \sum_{A \in T^{-m^{\prime} k} \mathcal{A}^{m}}|\rho(D, A)| \\
& \leq c_{8} r \psi(\Delta)
\end{aligned}
$$


by the mixing property if we assume $n$ is large enough.

(C) In order to apply Proposition 18 let us now define an interchangeable pair in the usual way by setting $V_{n}^{\prime}=V_{n}-\frac{1}{\sqrt{r}} X_{Y}+\frac{1}{\sqrt{r}} X^{*}$ where $Y \in\{0,1, \ldots, r-1\}$ is a randomly chosen index and $X^{*}$ is a random variable which is independent of all other random variables and has the same distribution as the $X_{j}$. Since the random variables $X_{j}$ for $j=0,1, \ldots, r-1$, are i.i.d., the pair $\left(V_{n}^{\prime}, V_{n}\right)$ is exchangeable. Moreover

$$
\mathbb{E}\left(V_{n}^{\prime} \mid V_{n}\right)=\left(1-\frac{1}{r}\right) V_{n}
$$

(i.e. $\lambda=\frac{1}{r}$ ).

We now estimate the two terms on the rights hand side of Proposition 18 separately:

(i) The third moment term of Proposition 18 is estimated using Corollary 16.

$$
\mathbb{E}\left(\left|V_{n}^{\prime}-V_{n}\right|^{3}\right)^{\frac{1}{3}}=\frac{1}{\sqrt{r} \sigma_{m}} \mathbb{E}\left(\left|J_{m} \circ T^{m^{\prime} Y}+J_{m}^{*}\right|^{3}\right)^{\frac{1}{3}} \leq \frac{2}{\sqrt{r} \sigma_{m}} M_{3}\left(J_{m}^{3}\right)^{\frac{1}{3}} \leq \frac{c_{9}}{\sqrt{r}} .
$$

Hence

$$
\sqrt{\frac{1}{\lambda} \mathbb{E}\left(\left|V_{n}^{\prime}-V_{n}\right|^{3}\right)}=\sqrt{\frac{c_{9}^{3} r}{r^{\frac{3}{2}}}}=\mathcal{O}\left(r^{-\frac{1}{4}}\right) .
$$

(ii) To estimate the variance term we follow Stein [37] and obtain

$$
\operatorname{var}\left(\mathbb{E}\left(\left(V_{n}^{\prime}-V_{n}\right)^{2} \mid V_{n}\right)\right) \leq \frac{1}{r^{2}} \operatorname{var}\left(\left(X_{Y}-X^{*}\right)^{2} \mid X_{0}, X_{1}, \ldots, X_{r-1}\right) .
$$

Since

we get

$$
\mathbb{E}\left(X_{j}^{2} \mid V_{n}\right)=\frac{1}{r} \sum_{i} \mathbb{E}\left(X_{i}^{2} \mid V_{n}\right)=\frac{1}{r} \sum_{i} X_{i}^{2}
$$

$$
\operatorname{var}\left(\mathbb{E}\left(X_{Y}^{2} \mid V_{n}\right)\right)=\operatorname{var}\left(\frac{1}{r} \sum_{i} X_{i}^{2}\right)=\frac{1}{r^{2}} \operatorname{var}\left(\sum_{i} X_{i}^{2}\right)=\frac{1}{r^{2}} r \operatorname{var}\left(X_{0}^{2}\right)=\frac{1}{r} \operatorname{var}\left(X_{0}^{2}\right) .
$$

Since $X_{0}$ has the same distribution as $\frac{1}{\sigma_{m}} J_{m}$ we have $\mathbb{E}\left(X_{0}\right)=0$ and by Propositions 14 and 15

$$
\operatorname{var}\left(X_{0}^{2}\right)=\operatorname{var}\left(\frac{1}{\sigma_{m}^{2}} J_{m}^{2}\right)=\frac{1}{\sigma_{m}^{4}} \sigma^{2}\left(J_{m}^{2}\right) \leq \frac{1}{\sigma_{m}^{4}} M_{4}\left(\mathcal{A}^{m}\right) \leq c_{10}
$$

Hence

$$
\frac{6}{\lambda} \sqrt{\operatorname{var}\left(\mathbb{E}\left(\left(V_{n}^{\prime}-V_{n}\right)^{2} \mid V_{n}\right)\right)} \leq c_{11} r \sqrt{\frac{1}{r^{3}}} \leq c_{11} \frac{1}{\sqrt{r}}
$$

Combining the estimates (i) and (ii) yields by Proposition 18

$$
\left|\mathbb{P}\left(V_{n} \leq t\right)-N(t)\right| \leq c_{11} \frac{1}{\sqrt{r}}+\frac{6 \sqrt{c_{9}}}{r^{\frac{1}{4}}} \leq c_{12} \frac{1}{\sqrt[4]{r}} .
$$

(D) Part (B) and (C) combined yield

$$
\left|\mathbb{P}\left(\hat{W}_{n} \leq t\right)-N(t)\right| \leq\left|\mathbb{P}\left(V_{n} \leq t\right)-N(t)\right|+\left\|D_{\hat{W}_{n}}-D_{V_{n}}\right\|_{\infty} \leq c_{12} \frac{1}{\sqrt[4]{r}}+c_{8} r \psi(\Delta) .
$$

Let us put $\epsilon=\left\|W_{n}-\hat{W}_{n}\right\|_{2}$ and $\epsilon^{\prime}=\sup _{t}\left|\mathbb{P}\left(\hat{W}_{n} \leq t\right)-N(t)\right|$. Then $\left(D_{W_{n}}\right.$ is the distribution function of $\left.W_{n}\right) N(t) \leq \epsilon^{\prime}$ for $t \leq-\left|\log \epsilon^{\prime}\right|$ and therefore $D_{\hat{W}_{n}}(t) \leq 2 \epsilon^{\prime}$ for $t \leq-\left|\log \epsilon^{\prime}\right|$ and similarly $|1-N(t)| \leq \epsilon^{\prime}$ and consequently $\left|1-D_{\hat{W}_{n}}(t)\right| \leq 2 \epsilon^{\prime}$ for all $t \geq\left|\log \epsilon^{\prime}\right|$ we get

$$
\left\|\left(D_{W_{n}}-D_{\hat{W}_{n}}\right) \chi_{\left[-\left|\log \epsilon^{\prime}\right|,\left|\log \epsilon^{\prime}\right|\right]}\right\|_{\infty} \leq 2\left|\log \epsilon^{\prime}\right| \cdot\left\|W_{n}-\hat{W}_{n}\right\|_{2}=2\left|\log \epsilon^{\prime}\right| \epsilon
$$


and (since distribution functions are increasing)

$$
\left\|D_{W_{n}}-D_{\hat{W}_{n}}\right\|_{\infty} \leq 2\left|\log \epsilon^{\prime}\right| \epsilon+2 \epsilon^{\prime}
$$

(E) To optimise the bound

$$
\left|\mathbb{P}\left(W_{n} \leq t\right)-N(t)\right| \leq\left|\mathbb{P}\left(\hat{W}_{n} \leq t\right)-N(t)\right|+\left\|D_{W_{n}}-D_{\hat{W}_{n}}\right\|_{\infty} \leq 2\left|\log \epsilon^{\prime}\right| \epsilon+3 \epsilon^{\prime}
$$

we distinguish between the case when $\psi$ decays (i) polynomially and (ii) hyper polynomially.

(i) Assume that $\psi$ decays polynomially with power $p>12$. Let $\delta, \alpha \in(0,1)$ and put $m=\left[n^{\alpha}\right], \Delta=\left[m^{\delta}\right]$ (i.e. $\Delta \sim n^{\alpha \delta}, \psi(\Delta)=\mathcal{O}\left(n^{-\alpha \delta p}\right)$. Then (assuming $n^{\frac{1}{2} \beta} \sqrt{\psi(\Delta)}=\mathcal{O}(1)$ which will be satisfied once we choose $\beta$ and $\delta$ )

$$
\left\|\hat{W}_{n}-W_{n}\right\|_{2} \leq c_{13}\left(n^{\frac{1}{2}-\alpha+\alpha \delta}+n^{\frac{1}{2}-\alpha+\frac{3}{2} \beta-\frac{1}{2} \alpha \delta p}+n^{\frac{1}{2}+\beta-\alpha-\frac{1}{2}(\beta-1) w}+n^{\frac{1}{2}-\frac{\alpha}{2}-\alpha \eta}\right) .
$$

The first three terms on the right hand side are optimised by $\beta=\frac{w(p+2)}{(p+2)(w-2)+6}$ and $\alpha \delta=\frac{3 \beta}{p+2}$. Then $\left\|\hat{W}_{n}-W_{n}\right\|_{2} \leq \epsilon, \epsilon=\mathcal{O}\left(n^{x}\right)$, where $x=\max \left(\frac{1}{2}-\alpha+\frac{3 w}{(p+2)(w-2)+6}, \frac{1}{2}-\frac{\alpha}{2}-\alpha \eta\right)$. The fourth term is smaller than the first three since we can assume that $\eta>\frac{1}{3}$ as $w>4$. The value of $\alpha$ is found by minimising the error term $2 \epsilon\left|\log \epsilon^{\prime}\right|+3 \epsilon^{\prime}$. Ignoring the logarithmic term we obtain $\alpha=\frac{3}{5}+\frac{12}{5} \frac{w}{(p+2)(w-2)+6}$ which implies

$$
\left|\mathbb{P}\left(W_{n} \leq t\right)-N(t)\right| \leq c_{14} \frac{1}{n^{\kappa}},
$$

for any $\kappa<\frac{1}{10}-\frac{3}{5} \frac{w}{(p+2)(w-2)+6}$. Note that $\alpha \eta>\kappa$ for all (possible) values of $p$ and $w$.

(ii) If $\psi$ decays faster than any power then we can choose $\delta>0$ arbitrarily close to zero and obtain $\alpha<\frac{3}{5}$ which yields the estimate $\left|\mathbb{P}\left(W_{n} \leq t\right)-N(t)\right| \leq c_{15} \frac{1}{n^{\kappa}}$, for any $\kappa<\frac{1}{10}$.

This concludes the proof since $W_{n}=\frac{I_{n}-H_{n}}{\sigma_{n}}$.

Proof of Theorem 2, We use Theorem 17 and have to make the following adjustments:

(i) To adjust for the difference between $H_{n}$ and $n h$ we use Lemma 13

$$
\mathbb{P}\left(\frac{I_{n}(x)-n h}{\sigma \sqrt{n}} \leq t\right)=\mathbb{P}\left(\frac{I_{n}(x)-H_{n}}{\sigma \sqrt{n}} \leq t+\mathcal{O}\left(n^{\frac{1}{2}-\gamma}\right)\right)=N(t)+\mathcal{O}\left(n^{-\kappa}\right)+\mathcal{O}\left(n^{\frac{1}{2}-\gamma}\right) .
$$

Since $p$ is big enough $\gamma$ can be chosen so that $\gamma-\frac{1}{2}>\kappa$.

(ii) By Proposition $14 \frac{\sigma_{n}}{\sqrt{n}}=\sigma+\mathcal{O}\left(n^{-\eta}\right)$ which yields

$$
\mathbb{P}\left(\frac{I_{n}(x)-H_{n}}{\sigma \sqrt{n}} \leq t\right)=\mathbb{P}\left(\frac{I_{n}(x)-H_{n}}{\sigma_{n}} \leq t_{n}\right)=N\left(t_{n}\right)+\mathcal{O}\left(n^{-\kappa}\right)=N(t)+\mathcal{O}\left(n^{-\min (\eta, \kappa)}\right),
$$

where $t_{n}=t \frac{\sigma \sqrt{n}}{\sigma_{n}}=t\left(1+\mathcal{O}\left(n^{-\eta}\right)\right)$. This concludes the proof since $\eta$ can be taken to be $>\kappa$.

\section{Proof of Theorem 5 (Weak Invariance Principle)}

In order to prove the WIP for $I_{n}(x)=-\log \mu\left(A_{n}(x)\right)$ denote by $W_{n, x}(t), t \in[0,1]$, its interpolation

$$
W_{n, x}(k / n)=\frac{I_{k}(x)-k h}{\sigma \sqrt{n}}
$$

$x \in \Omega$ and linearly interpolated on each of the subintervals $\left[\frac{k}{n}, \frac{k+1}{n}\right]$. In particular $W_{n, x} \in C_{\infty}([0,1])$ (with supremum norm). Denote by $D_{n}$ the distribution of $W_{n, x}$ on $C_{\infty}([0,1])$, namely

$$
D_{n}(H)=\mu\left(\left\{x \in \Omega: W_{n, x} \in H\right\}\right)
$$


where $H$ is a Borel subset of $C_{\infty}([0,1])$. The WIP then asserts that the distribution $D_{n}$ converges weakly to the Wiener measure, which means that $S_{n}=I_{n}-n h$ is for large $n$, and after a suitable normalization distributed approximately as the position at time $t=1$ of a particle in Brownian motion [3].

If we put $S_{i}=-\log \mu\left(A_{i}(x)\right)-i h(\mu)$ then two conditions have to be verified (3] Theorem 8.1), namely (A) The tightness condition: There exists a $\lambda>0$ so that for every $\varepsilon>0$ there exists an $N_{0}$ so that

$$
\mathbb{P}\left(\max _{0 \leq i \leq n}\left|S_{i}\right|>2 \lambda \sqrt{n}\right) \leq \frac{\varepsilon}{\lambda^{2}}
$$

for all $n \geq N_{0}$.

(B) The finite-dimensional distributions of $S_{i}$ converge to those of the Wiener measure.

(A) Proof of tightness: As before let $J_{i}=I_{i}-H_{i}$ and note that $i h-H_{i}=\mathcal{O}\left(i^{1-\gamma}\right), 1-\gamma \in\left(\frac{2 w}{p(w-1)}, 1\right)$, (Lemma 13) is easily absorbed by the term $\lambda \sqrt{n}$ as $1-\gamma<\frac{1}{2}$. In the usual way (cf. e.g. [3]) we get

$$
\mathbb{P}\left(\max _{0 \leq i \leq n}\left|J_{i}\right|>2 \lambda \sqrt{n}\right) \leq \mathbb{P}\left(\left|J_{n}\right|>\lambda \sqrt{n}\right)+\sum_{i=0}^{n-1} \mu\left(E_{i} \cap\left\{\left|J_{i}-J_{n}\right| \geq \lambda \sqrt{n}\right\}\right),
$$

where $E_{i}$ is the set of points $x$ so that $\left|J_{i}(x)\right|>2 \lambda \sqrt{n}$ and $\left|J_{k}(x)\right| \leq 2 \lambda \sqrt{n}$ for $k=0, \ldots, i-1$. Note that $E_{i}$ lies in the $\sigma$-algebra generated by $\mathcal{A}^{i}$. Clearly the sets $E_{i}$ are pairwise disjoint. To estimate $\mu\left(E_{i} \cap\left\{\left|J_{i}-J_{n}\right| \geq \lambda \sqrt{n}\right\}\right)$ let us first 'open a gap' of length $\Delta<\frac{n}{2}$. Let $\tilde{\mathcal{A}}^{n}=\mathcal{A}^{i} \vee T^{-i-\Delta} \mathcal{A}^{n-i-\Delta}$ (if $i<\frac{n}{2}$ and $\tilde{\mathcal{A}}^{n}=\mathcal{A}^{i-\Delta} \vee T^{-i} \mathcal{A}^{n-\Delta}$ if $\left.i \geq \frac{n}{2}\right)$, denote by $\tilde{I}_{n}$ its information function and by $\tilde{H}_{n}=\mu\left(\tilde{I}_{n}\right)$ its entropy. Obviously $H_{n} \geq \tilde{H}_{n}$ and moreover $\mu\left(I_{n}-\tilde{I}_{n}\right)=H_{n}-\tilde{H}_{n} \leq H_{\Delta} \leq c_{1} \Delta$. Since by Lemma 10 and Corollary 9 (as $\mathcal{A}^{n}$ refines $\tilde{\mathcal{A}}^{n}$ )

$$
\sigma\left(I_{n}-\tilde{I}_{n}\right)=\sigma\left(\mathcal{A}^{n} \mid \tilde{\mathcal{A}}^{n}\right) \leq \sqrt{K_{2}\left(\mathcal{A}^{\Delta}\right)} \leq c_{2} \Delta
$$

we obtain by Chebycheff's inequality $\left(\tilde{J}_{n}=\tilde{I}_{n}-\tilde{H}_{n}\right)$

$$
\mathbb{P}\left(\left|J_{n}-\tilde{J}_{n}\right| \geq \ell\right) \leq \frac{\sigma^{2}\left(I_{n}-\tilde{I}_{n}\right)}{\ell^{2}} \leq c_{3} \frac{\Delta^{2}}{\ell^{2}} .
$$

By the uniform strong mixing property

$$
\tilde{I}_{n}(B)=I_{i}(B)+I_{n-i-\Delta}(C)-\log \left(1+\frac{\rho(B, C)}{\mu(B) \mu(C)}\right)
$$

for all $(B, C) \in \mathcal{A}^{i} \times T^{-i-\Delta} \mathcal{A}^{n-i-\Delta}$. If $Y$ denotes the random variable on $\mathcal{A}^{i} \times T^{-i-\Delta} \mathcal{A}^{n-i-\Delta}$ whose values are $Y(B, C)=-\log \left(1+\frac{\rho(B, C)}{\mu(B) \mu(C)}\right)$ then by Lemma 11] $(a=2)$

$$
\sigma^{2}(Y) \leq\|Y\|_{L^{2}}^{2} \leq C_{4}\left(\psi(\Delta)(n-\Delta)^{3 \beta}+(n-\Delta)^{2 \beta-(\beta-1) w}\right)
$$

for $\beta>1$ arbitrary. By Chebycheff's inequality this implies

$$
\mathbb{P}\left(\left|\tilde{J}_{n}-J_{i}-J_{n-i-\Delta} \circ T^{i+\Delta}\right| \geq \ell\right) \leq \frac{\sigma^{2}(Y)}{\ell^{2}} \leq C_{4} \frac{\psi(\Delta)(n-\Delta)^{3 \beta}+n^{2 \beta-(\beta-1) w}}{\ell^{2}} .
$$

Then

$$
\begin{gathered}
\mu\left(E_{i} \cap\left\{\left|J_{n}-J_{i}\right| \geq \lambda \sqrt{n}\right\}\right) \leq \mu\left(E_{i} \cap\left\{\left|J_{n}-\tilde{J}_{n}\right| \geq \ell\right\}\right)+\mu\left(E_{i} \cap\left\{\left|\tilde{J}_{n}-J_{i}-J_{n-i-\Delta} \circ T^{i+\Delta}\right| \geq \ell\right\}\right) \\
+\mu\left(E_{i} \cap\left\{\left|J_{n-i-\Delta} \circ T^{i+\Delta}\right| \geq \lambda \sqrt{n}-2 \ell\right\}\right) .
\end{gathered}
$$


The last term on the right hand side can be estimated using the mixing property (note that $E_{i}$ is in the $\sigma$-algebra generated by $\mathcal{A}^{i}$, and $\left\{\left|J_{n-i-\Delta}\right| \geq \lambda \sqrt{n}-2 \ell\right\}$ is in the $\sigma$-algebra generated by $T^{-i-\Delta} \mathcal{A}^{n-i-\Delta}$ )

$$
\begin{aligned}
\mu\left(E_{i} \cap\left\{\left|J_{n-i-\Delta} \circ T^{i+\Delta}\right| \geq \lambda \sqrt{n}-2 \ell\right\}\right) & =\mu\left(E_{i}\right) \mathbb{P}\left(\left|J_{n-i-\Delta}\right| \geq \lambda \sqrt{n}-2 \ell\right) \\
& +\sum_{B \subset E_{i}} \sum_{C \subset T^{-i-\Delta}\left\{\left|J_{n-i-\Delta}\right| \geq \lambda \sqrt{n}-2 \ell\right\}} \rho(B, C) \\
& \leq \mu\left(E_{i}\right)\left(2 N\left(\frac{\lambda \sqrt{n}-2 \ell}{\sigma_{n-i-\Delta}}\right)+C_{0}(n-i-\Delta)^{-\kappa}\right)+\psi(\Delta)
\end{aligned}
$$

using Theorem 17 in the last step.

We finally obtain (as $\left.\mathbb{P}\left(\left|J_{n}\right|>\lambda \sqrt{n}\right) \leq 2 N(\lambda)+c_{4} n^{-\kappa}\right)$

$$
\begin{aligned}
\mathbb{P}\left(\max _{0 \leq i \leq n}\left|J_{i}\right|>2 \lambda \sqrt{n}\right) \leq & 2 N(\lambda)+c_{4} n^{-\kappa}+\sum_{i} \mu\left(E_{i} \cap\left\{\left|J_{n}-\tilde{J}_{n}\right| \geq \ell\right\}\right) \\
& +n C_{4} \frac{\psi(\Delta) n^{3 \beta}+n^{2 \beta-(\beta-1) w}}{\ell^{2}} \\
& +\sum_{i} \mu\left(E_{i}\right)\left(2 N\left(\frac{\lambda \sqrt{n}-2 \ell}{\sigma_{n-i-\Delta}}\right)+C_{0}(n-i-\Delta)^{-\kappa}\right)+n \psi(\Delta) \\
\leq & 2 N(\lambda)+c_{5} n^{-\kappa}+c_{6} \frac{\Delta^{2}+\psi(\Delta) n^{3 \beta}+n^{2 \beta-(\beta-1) w}}{\ell^{2}}+2 N\left(\frac{\lambda \sqrt{n}-2 \ell}{\sqrt{n}}\right)
\end{aligned}
$$

(if $\Delta<\frac{n}{2}$ is small enough). If $\psi$ decays at least polynomially with a power larger than $8+\frac{24}{w-4}$ then we can put $\ell \sim n^{\alpha}, \Delta \sim n^{\alpha^{\prime}}$ and choose $\alpha^{\prime}<\alpha<\frac{1}{2}$ and $\beta>1$ (e.g. $\beta=\frac{w}{w-2}, \alpha^{\prime}<\frac{3 \beta}{p}$ ) so that the terms on the right hand side which don't involve the normal probability $N$ decay polynomially in $n$. This proves the tightness condition (3), since for every $\varepsilon>0$ one can find a $\lambda>1$ so that the quadratic estimate holds for all $n$ large enough.

(B) Proof of the finite-dimensional distribution convergence: For $t \in[0,1]$ define the random variable

$$
X_{n}(t, x)=\frac{1}{\sigma \sqrt{n}}\left(S_{[n t]}(x)+(n t-[n t])\left(S_{[n t]+1}(x)-S_{[n t]}(x)\right)\right)
$$

which interpolates $S_{[n t]}$. It is defined on $\Omega$ and has values in $C_{\infty}([0,1])$.

We must show that the distribution of $\left(X_{n}(t, x), X_{n}(t, x)-X_{n}(s, x)\right)$ converges to $(\mathcal{N}(0, t), \mathcal{N}(0, t-s))$ $(0 \leq s<t)$ as $n \rightarrow \infty$, where $\mathcal{N}(0, t)$ is the normal distribution with zero mean and variance $t^{2}$. To prove this as well as the convergence of higher finite dimensional distributions it suffices to show that $X_{n}(t, x)-X_{n}(s, x)$ converges to $\mathcal{N}(0, t-s)$ ([3] Theorem 3.2). We obtain by Lemma 13

$$
S_{[n t]}-S_{[n s]}=J_{[n t]}-J_{[n s]}+\mathcal{O}\left((n t)^{1-\gamma}\right)
$$

and by (4), (5) and Theorem 2

$$
\begin{aligned}
& \mathbb{P}\left(\frac{S_{[n t]}-S_{[n s]}}{\sigma \sqrt{n}} \geq \lambda\right) \leq \mathbb{P}\left(\left|J_{[n t]}-\tilde{J}_{[n t]}\right| \geq \ell\right)+\mathbb{P}\left(\left|\tilde{J}_{[n t]}-J_{[n s]}-J_{[n t]-[n s]-\Delta} \circ T^{[n s]+\Delta}\right| \geq \ell\right) \\
& +\mathbb{P}\left(\left|J_{[n t]-[n s]-\Delta}\right| \geq \lambda \sigma \sqrt{n}-2 \ell\right)+\mathcal{O}\left((n t)^{\frac{1}{2}-\gamma}\right) \\
& \leq \frac{\sigma^{2}\left(I_{n}-\tilde{I}_{n}\right)}{\ell^{2}}+\frac{\sigma^{2}(Y)}{\ell^{2}}+N\left(\frac{\lambda \sigma \sqrt{n}-2 \ell}{\sqrt{[n t]-[n s]-\Delta}}\right)+\frac{C_{0}}{([n t]-[n s]-\Delta)^{\kappa}}+\frac{\mathcal{O}(1)}{(n t)^{1-\gamma}} \\
& \leq \quad c_{3} \frac{\Delta^{2}}{\ell^{2}}+C_{4} \frac{\psi(\Delta)(n t)^{3 \beta}+(n t)^{2 \beta-(\beta-1) w}}{\ell^{2}}+\frac{c_{7}}{(n(t-s))^{\kappa}}+N\left(\frac{\lambda}{\sqrt{t-s}}\right) \text {, }
\end{aligned}
$$


assuming $\frac{1}{2}-\gamma \geq \kappa$ and $n(t-s) \gg \Delta$. Similarly to above we used a random variable $Y$ on $\mathcal{A}^{[n s]} \times$ $T^{-[n s]-\Delta} \mathcal{A}^{[n t]-[n s]-\Delta}$ given by $Y(B, C)=-\log \left(1+\frac{\rho(B, C)}{\mu(B) \mu(C)}\right)$. Now let $\ell \sim n^{\alpha}, \Delta \sim n^{\alpha^{\prime}}$ and $\alpha^{\prime}<\alpha<\frac{1}{2}$ and $\beta>1$ so that the terms on the right hand side other than $N(\lambda / \sqrt{t-s})$ decay polynomially in $n$. Hence $S_{[n t]}-S_{[n s]}$ and therefore $X_{n}(t, x)-X_{n}(s, x)$ converges in distribution to $\mathcal{N}(0, \sqrt{t-s})$ as $n \rightarrow \infty$.

\section{Appendix (Markov chains)}

Here we compute the variance for the Markov measure on an infinite alphabet. As in section 2.3 let $\Sigma$ be the shiftspace over the alphabet $\mathbb{N}$ and $\mu$ the Markov measure generated by the probability vector $\vec{p}$ and stochastic matrix $P$. Then

$$
\sigma_{n}^{2}=\frac{1}{2} \sum_{\vec{x}, \vec{y} \in \mathcal{A}^{n}} \mu(\vec{x}) \mu(\vec{y})\left(\log \frac{p_{x_{1}}}{p_{y_{1}}}+\sum_{j=1}^{n-1} \log \frac{P_{x_{j} x_{j+1}}}{P_{y_{j} y_{j+1}}}\right)^{2}=A+B+C+D,
$$

where

$$
A=\frac{1}{2} \sum_{\vec{x}, \vec{y} \in \mathcal{A}^{n}} \mu(\vec{x}) \mu(\vec{y}) \log ^{2} \frac{p_{x_{1}}}{p_{y_{1}}}=\frac{1}{2} \sum_{i j} p_{i} p_{j} \log ^{2} \frac{p_{i}}{p_{j}}=\mathcal{O}(1)
$$

and

$$
\begin{aligned}
B & =\sum_{j=1}^{n-1} \sum_{\vec{x}, \vec{y} \in \mathcal{A}^{n}} \mu(\vec{x}) \mu(\vec{y}) \log \frac{p_{x_{1}}}{p_{y_{1}}} \log \frac{P_{x_{j} x_{j+1}}}{P_{y_{j} y_{j+1}}} \\
& =\sum_{j=1}^{n-1} \sum_{\vec{x}, \vec{y} \in \mathcal{A}^{j+1}} \mu(\vec{x}) \mu(\vec{y})\left(\log p_{x_{1}} \log P_{x_{j} x_{j+1}}+\log p_{y_{1}} \log P_{y_{j} y_{j+1}}-\log p_{x_{1}} \log P_{y_{j} y_{j+1}}-\log p_{y_{1}} \log P_{x_{j} x_{j+1}}\right) \\
& =2 \sum_{j=1}^{n-1} \sum_{\vec{x} \in \mathcal{A}^{j+1}} \mu(\vec{x}) \log p_{x_{1}} \log P_{x_{j} x_{j+1}}+2(n-1) h \sum_{i} p_{i} \log p_{i} .
\end{aligned}
$$

Since Markov chains are exponentially mixing [4] we get for some $\vartheta \in(0,1)$ that

$$
\sum_{\vec{x} \in \mathcal{A}^{j+1}} \mu(\vec{x}) \log p_{x_{1}} \log P_{x_{j} x_{j+1}}=\sum_{i} p_{i} \log p_{i} \sum_{i j} p_{i} P_{i j} \log P_{i j}+\mathcal{O}\left(\vartheta^{j}\right)=-h \sum_{i} p_{i} \log p_{i}+\mathcal{O}\left(\vartheta^{j}\right)
$$

and therefore

$$
B=2 \sum_{j} \mathcal{O}\left(\vartheta^{j}\right)=\mathcal{O}(1)
$$

The principal term is

$$
D=\frac{1}{2} \sum_{j=1}^{n-1} \sum_{\vec{x}, \vec{y} \in \mathcal{A}^{n}} \mu(\vec{x}) \mu(\vec{y}) \log ^{2} \frac{P_{x_{j} x_{j+1}}}{P_{y_{j} y_{j+1}}}=\frac{n-1}{2} \sum_{i j k \ell} p_{i} P_{i j} p_{k} P_{k \ell} \log ^{2} \frac{P_{i j}}{P_{k \ell}} .
$$

Lastly we get the correction term

$$
\begin{aligned}
C & =\sum_{i \neq j} \sum_{\vec{x}, \vec{y} \in \mathcal{A}^{n}} \mu(\vec{x}) \mu(\vec{y}) \log \frac{P_{x_{i} x_{i+1}}}{P_{y_{i} y_{i+1}}} \log \frac{P_{x_{j} x_{j+1}}}{P_{y_{j} y_{j+1}}} \\
& =2 \sum_{k=1}^{n-1}(n-k) \sum_{\vec{x}, \vec{y} \in \mathcal{A}^{k+1}} \mu(\vec{x}) \mu(\vec{y}) \log \frac{P_{x_{1} x_{2}}}{P_{y_{1} y_{2}}} \log \frac{P_{x_{k} x_{k+1}}}{P_{y_{k} y_{k+1}}}
\end{aligned}
$$




$$
\begin{aligned}
& =2 \sum_{k=1}^{n-1}(n-k) \sum_{\vec{x}, \vec{y} \in \mathcal{A}^{k+1}} \mu(\vec{x}) \mu(\vec{y})\left(\log P_{x_{1} x_{2}} \log P_{x_{k} x_{k+1}}+\log P_{y_{1} y_{2}} \log P_{y_{k} y_{k+1}}-\log P_{x_{1} x_{2}} \log P_{y_{k} y_{k+1}}-\log P_{y_{1} y_{2}} \log \right. \\
& =4 \sum_{k=1}^{n-1}(n-k)\left(\sum_{\vec{x} \in \mathcal{A}^{k+1}} \mu(\vec{x}) \log P_{x_{1} x_{2}} \log P_{x_{k} x_{k+1}}-h^{2}\right)
\end{aligned}
$$

Since $\sigma^{2}=\lim _{n \rightarrow \infty} \frac{\sigma_{n}^{2}}{n}$ we finally obtain

$$
\sigma^{2}=\frac{1}{2} \sum_{i j k \ell} p_{i} P_{i j} p_{k} P_{k \ell} \log ^{2} \frac{P_{i j}}{P_{k \ell}}+4 \sum_{k=1}^{\infty} \sum_{\vec{x} \in \mathcal{A}^{k+1}} \mu(\vec{x})\left(\log P_{x_{1} x_{2}} \log P_{x_{k} x_{k+1}}-h^{2}\right),
$$

where the infinite sum converges because the terms (correlations) decay exponentially fast.

\section{References}

[1] M Abadi: Exponential Approximation for Hitting Times in Mixing Stochastic Processes; Mathematical Physics Electronic Journal 7 (2001)

[2] S Bernstein: Sur l'extension du théorème limite du calcul des probabilités aux sommes du quantités dépendantes; Math. Ann. 97 (1926), 1-59

[3] P Billingsley: Convergence in Probability Measures; Wiley 1968

[4] X Bressaud: Subshifts on an infinite alphabet; Ergod. Th. \& Dynam. Sys. (1999) 19, 1175-1200

[5] M Brin and A Katok: On local entropy; Geometric Dynamics 30-38, Springer LNM \#1007, 1983

[6] A Broise: Transformations dilatantes de l'intervalle et théorèmes limites; Asterisque \#238, 1996

[7] H Bruin and S Vaienti: Return times for unimodal maps; submitted to Forum Math.

[8] L Carleson: Two remarks on the basic theorem of information theory; Math. Scand. 6 (1958), $175-180$

[9] N Chernov: Limit theorems and Markov approximations for chaotic dynamical systems; Prob. Th. Rel. Fields 101 (1995), 321-362

[10] K L Chung: A note on the ergodic theorem of information theory; Ann. Math. Stat. 32 (1961), 612-614

[11] P Collet and A Galves: Repetition times for Gibbsian sources; Nonlinearity 12 (1999), 1225-1237

[12] P Collet, A Galves and B Schmitt: Fluctuations of repetition times for Gibbsian sources; Nonlinearity 12 (1999), 1225-1237

[13] P Doukhan: Mixing: Properties and examples; Lecture Notes in Statistics 85, Springer 1995

[14] P Ferrero, N Haydn and S Vaienti: Entropy fluctuations for parabolic maps; Nonlinearity 16 (2003), $1203-1218$

[15] M Field, I Melbourne and A Török: Decay of Correlations, Central Limit Theorems and Approximations by Brownian Motion for Compact Lie Group Extensions; Ergod. Th. \& Dynam. Syst. 23 (2003), 87-110

[16] A Galves and B Schmitt: Inequalities for hitting times in mixing dynamical systems; Random and Computational Dynamics 5 (1997), 337-348 
[17] M Gordin: The central limit theorem for stationary processes; Soviet Math. Doklady 10 (1969), $1174-1176$

[18] N Haydn and S Vaienti: Fluctuations of the metric entropy for mixing measures; Stochastics and Dynamics 4 (2004), 595-627

[19] N Haydn and S Vaienti: The distribution of the measure of cylinders for non-Gibbsian measures; Complex Dynamics and Related Topics 147-162, New Studies in Advanced Mathematics \#5, 2003

[20] I A Ibragimov: Some limit theorems for stationary processes; Theory Prob. Appl. 7 (1962), 349-382

[21] I Kontoyiannis: Asymptotic Recurrence and Waiting Times for Stationary Processes; J. Theor. Prob. 11 (1998), 795-811

[22] C Liverani: Central Limit theorem for deterministic systems; Intern. Congress on Dyn. Syst., Montevideo 1995 (Proc. Research Notes in Math. Series), Pitman (1996), 56-75

[23] R Mañé: Ergodic Theory and Differentiable Dynamics, Springer 1987

[24] S Nagaev: Some limit theorems for stationary Markov chains; Theory Prob. Appl. 2 (1957), 378-406

[25] A Nobel and A Wyner: A Recurrence Theorem for Dependent Processes with Applications to Data Compression; IEEE Vol. 38 No. 5 (1992), 1561-1564

[26] Ornstein and Weiss; Entropy and Data Compression Schemes; IEEE Transactions on Information Theory 39 (1993), 78-83

[27] Ornstein and Weiss; Entropy and Recurrence Rates for Stationary Random Fields; IEEE Transactions on Information Theory 48 (6) (2002), 1694-97

[28] F Paccaut: Propriétés Statistiques de Systèmes Dynamiques Non Markovian; Thèse (Doctorat) Dijon 2000

[29] F Pène: Rates of Convergence in the CLT for Two-Dimensional Dispersive Billiards; Commun. Math. Phys. 225 (2002), 91-119

[30] K Petersen: Ergodic Theory: Cambridge studies in advanced mathematics \#2, 1983

[31] V V Petrov: On a relation between an estimate of the remainder in the central limit theorem and the law of the iterated logarithm; Teor. Veroyatn. Primen. 11 (3), 514-518. English translation: Theor. Probab. Appl. 11(3), 454-458.

[32] W Philipp and W Stout: Almost sure invariance principles for partial sums of weakly dependent random variables; AMS Memoirs vol. 2 issue 2 No. 161, 1975

[33] Y Rinott and V Rotar: Normal approximations by Stein's method; Decis. Econ. Finance 23 (2000), 15-29

[34] V I Romanovskii: Discrete Markov Chains; Wolters-Noordhoff Publishing Groningen 1970

[35] M Rosenblatt: A central limit theorem and a strong mixing condition; Proc. Nat. Acad. Sci. USA 42 (1956), 43-47

[36] M Rosenblatt: Markov Processes. Structure and Asymptotic Behavior; Springer, Grundlagen \#184, 1971

[37] C Stein: Approximate Computation of Expectations; IMS Lecture Notes \#7, 1986

[38] P Walters: An Introduction to Ergodic Theory; Springer-Verlag 1981 
[39] L-S Young: Statistical properties of dynamical systems with some hyperbolicity: Ann. of Math. 147 (1998), 585-650

[40] A Yushkevich: On limit theorems connected with the concept of the entropy of Markov chains (in Russian); Uspehi Math. Nauk 8 (1953) 177-180 